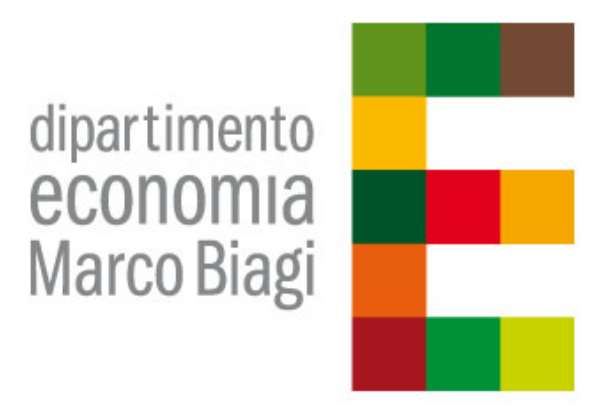

DEMB Working Paper Series

\author{
N. 89 \\ Sensitivity analysis and investment decisions: \\ NPV-consistency of rates of return \\ Andrea Marchioni*, Carlo Alberto Magni**
}

June 2016

* University of Modena and Reggio Emilia email: andrea.marchioni1989@gmail.com

** University of Modena and Reggio Emilia CEFIN - Center for Research in Banking and Finance Address: Viale Berengario 51, 41121 Modena, Italy email: magni@unimo.it

ISSN: 2281-440X online 


\title{
Sensitivity analysis and investment decisions: NPV-consistency of rates of return
}

\author{
Andrea Marchioni \\ Department of Economics "Marco Biagi" \\ University of Modena and Reggio Emilia \\ Carlo Alberto Magni \\ Department of Economics "Marco Biagi" \\ CEFIN - Center for Research in Banking and Finance \\ University of Modena and Reggio Emilia*
}

\begin{abstract}
Investment decisions may be evaluated via several different metrics, which are functions of a vector of value drivers. The economic significance and reliability of a metric depend on its consistency with the Net Present Value (NPV), which signals shareholder value creation. Traditionally, a metric is NPV-consistent if it correctly signals value creation. This paper introduces a new, stronger definition of NPV-consistency that takes into account the influence of value drivers on the metric output. A metric is strongly NPV-consistent if (it signals value creation and) the ranking of the value drivers in terms of impact on the output is the same as that provided by the NPV. We show that Internal Rate of Return (IRR) is not strongly NPV-consistent and show that its degree of inconsistency is not negligible by means of both Spearman's (1904) correlation coefficient and Iman and Conover's (1987) top-down coefficient. We introduce a new metric, called straight-line AIRR, belonging to the class of AIRRs (Magni 2010, 2013), which is associated with straight-line capital depreciation. This new metric enjoys strong NPV-consistency under several (possibly all) methods of sensitivity analysis.
\end{abstract}

Keywords. Sensitivity analysis, investment decisions, NPV, consistency, straight-line AIRR, IRR.

JEL codes. D81, M41, G31, G12, D92, Q28.

*Corresponding author: tel. +39-059-2056777, e-mail: magni@unimo.it 


\section{Introduction}

In capital budgeting many different criteria are used for evaluating a project and making decisions. Net Present Value (NPV) is considered the most theoretically reliable tool, since it correctly measures shareholder value creation (Brealey and Myers 2000, Ross, Westerfield and Jordan 2011). However, in practice, many other metrics are used, in particular, relative measures of worth such as rates of return. Among these, the Internal Rate of Return (IRR) is widely reported to be a common metric for measuring the economic profitability of a project. Recently, a more general notion of rate of return, labeled AIRR (Average Internal Rate of Return) has been developed by Magni (2010, 2013), based on a capital-weighted mean of holding period rates. The AIRR approach consists in associating the capital amounts invested in each period with the corresponding period returns by means of a weighted arithmetic mean. Magni $(2010,2013)$ showed that IRR itself is but a special case of AIRR associated with so-called Hotelling values. Magni (2010, 2013) showed that any AIRR is NPV-consistent in that decisions made by an investor who adopts NPV are the same as those made by an investor who adopts AIRR. However, this kind of coherence is necessary but not sufficient to make a rate of return a reliable evaluation and decision tool. Under uncertainty, the base (i.e. expected) value of an NPV or a rate of return is not the only element that drives a decision. The analysis of the robustness of a decision under changes in the inputs is important, as well as the investigation of the risk factors that mainly influence the value of the objective function.

Sensitivity analysis (SA) investigates the variation of an objective function under changes in the key inputs of a model, so aiming at identifying the most important risk factors affecting the objective function (and, therefore, the decision) and ranking them. There are many different SA techniques (see Pianosi et al. 2014, Borgonovo and Plischke 2016) and, given a technique, different objective functions may or may not lead to different results.

This paper applies SA to investment decisions. In this context, an objective function is a metric depending on a set of value drivers that aims at measuring the economic profitability (i.e., value created) of the project. We use different metrics in order to study their coherence and, more compellingly, the coherence with NPV. In particular, we first introduce a new rate of return, called straight-line AIRR (SL-AIRR). The SL-AIRR is associated with capital amounts that depreciate uniformly through time (straight-line depreciation). Hence, we compare NPV, IRR and SL-AIRR and analyze their reciprocal coherence. To this end, we give a new definition of NPV-consistency (strong coherence), according to which a metric is NPV-consistent under a given SA technique if it is NPVconsistent in the traditional sense and, in addition, the ranking of the project's value drivers (in terms of influence on the output) is the same. If a metric is not NPV-consistent, we measure the degree of inconsistency by means of two alternative indices: Spearman's (1904) coefficient or Iman and Conover's (1987) top-down coefficient.

We find that the SL-AIRR is more reliable than the IRR in more than one sense. First, we show that SL-AIRR is an affine transformation of NPV, which implies that it is strongly coherent with the NPV under many techniques, even in a strict sense (the relevances of the parameters are the same), whereas IRR is not strongly NPV-consistent and the degree of inconsistency may be remarkable. Furthermore, while IRR may not exist or may not be unique, the SL-AIRR always exists and is unique. Moreover, the IRR may exist and be unique if the base values of the key drivers are considered, whereas it may not exist or be multiple if other values of the key drivers are considered, so the case might occur where it is impossible to perform a sensitivity analysis. Finally, while the IRR may change its financial nature under changes in the cost of capital (investment rate versus financing rate), the SL-AIRR has an unambiguous financial nature determined by the sign of the first cash flow: An investment rate if the initial amount is negative, a financing rate if it is positive.

As a result, the use of IRR for investment evaluation should be discouraged. The SL- 
AIRR is a more reliable measure of worth, which can coherently be associated with NPV in investment evaluation and decisions. Indeed, the SL-AIRR even provides information that the traditional NPV analysis cannot provide. In particular, the SL-AIRR (i) supplies information about the return per unit of total capital committed, (ii) enables interpreting the project as an investment or a financing, and, therefore, whether value is created because funds are invested at a rate of return which is greater than the cost of capital (COC) or because funds are borrowed at financing rate which is smaller than the COC, (iii) decomposes the economic value created into economic efficiency (the difference between SL-AIRR and COC) and the investment scale (the sum of the committed amounts).

The remaining part of the paper is structured as follows. Section 2 introduces the SL-AIRR and describes its properties. Section 3 describes some well-known SA methods and Section 4 introduces the notion of pairwise coherence according to which any two functions are strongly coherent if the ranking of the model parameters coincides. This section shows that, under many SA techniques, a function $f$ and an affine transformation of it share the same (ranking and) relevances of parameters, so they are strongly coherent in a strict sense. Section 5 shows that the SL-AIRR is strongly NPV-consistent in a strict sense under many SA techniques, whereas IRR is incompatible with NPV. Some numerical counterexamples in section 6 show that even in very simple cases, the discrepancy between IRR and NPV may be not negligible and some problems of ambiguity may arise, which suggests that the use of IRR should be discouraged. Some concluding remarks end the paper.

\section{IRR and SL-AIRR as special cases of AIRR}

Let $P$ be a project and let $F=\left(F_{0}, F_{1}, \ldots, F_{p}\right), F \neq 0$, its estimated stream of free cash flows (FCFs), where $p$ is the lifetime of the project. Let $c_{t}$ be the capital invested (or borrowed, if negative) in the interval $[t, t+1]$. Then, the capital evolves recursively as

$$
c_{t}=c_{t-1}+I_{t}-F_{t}
$$

where $I_{t}$ is the net operating profit (after taxes). The boundary conditions are $F_{0}=-c_{0}$ and, after liquidation, $c_{p}=0$. The stream of capital amounts is then $c=\left(-F_{0}, c_{1}, \ldots, c_{p-1}, 0\right)$. In corporate projects, pro forma financial statements are available which report the base values of revenues, costs, interest and accruals (working capital, net fixed assets, debt). In particular, the capital can be decomposed into working capital $(W C)$ and net fixed assets $(N F A)$ and the following accounting identity holds in every period:

$$
N F A_{t}+W C_{t}=D_{t}+E_{t}
$$

where $D_{t}$ denotes net financial obligations and $E_{t}$ denotes equity. Let $R_{t}$ and $C_{t}$ be the revenues and the operating costs. Then, $I_{t}=\left(R_{t}-C_{t}-D e p_{t}\right)(1-\tau)$ where Dep $p_{t}$ is the depreciation charge for the fixed assets and $\tau$ is the company tax rate. Using (1), FCF is then derived as

$$
F_{t}=\left(R_{t}-C_{t}-D e p_{t}\right)(1-\tau)-\left(N F A_{t}-N F A_{t-1}\right)-\left(W C_{t}-W C_{t-1}\right) .
$$

Let $k$ be the (assumed constant) cost of capital (COC), that is, the minimum attractive rate of return (if $c_{t}>0$ ) or the maximum attractive financing rate (if $c_{t}<0$ ). We assume that the COC is exogenously fixed by the decision-maker/analyst. It is well-known that net present value (NPV) measures the economic value created: NPV $=\sum_{t=0}^{p} F_{t}(1+k)^{-t}$. Therefore, the NPV decision criterion may be stated as follows:

Definition 1. (NPV criterion) A project creates value (i.e., it is worth undertaking) if and only if the project $N P V$, computed at the discount rate $k$, is positive: $N P V(k)>0$. 
An AIRR, denoted as $\bar{\imath}$, is defined as the ratio of the overall income $I=\sum_{t=1}^{p} I_{t}(1+$ $k)^{-(t-1)}$ earned by the investor to the overall capital committed $C=\sum_{t=1}^{p} c_{t-1}(1+$ $k)^{-(t-1)}$ :

$$
\bar{\imath}=\frac{I}{C}
$$

or, equivalently, as the weighted mean of period rates associated with the capital stream $c$ :

$$
\bar{\imath}=\frac{\sum_{t=1}^{p} i_{t} c_{t-1}(1+k)^{-(t-1)}}{\sum_{t=1}^{p} c_{t-1}(1+k)^{-(t-1)}}
$$

where $i_{t}=I_{t} / c_{t-1}$ is the growth rate for capital (see Magni 2010, 2013).

Magni $(2010,2013)$ defined a project a net investment if $C>0$ and a net financing if $C<0$. In such a way, the financial nature of any project (and its associated rate of return) can be identified as an investment project or a financing project (respectively, an investment rate or a financing rate).

Traditionally, it is widely accepted that a metric/criterion $\varphi$ is to be NPV-consistent if and only if a decision maker adopting $\varphi$ makes the same decision suggested by the NPV criterion. We can formalize this standard notion as follows.

Definition 2. (NPV-consistency) A metric/criterion $\varphi$ is NPV-consistent if, given a cutoff rate $k$, the following statements are true:

(i) An investment project creates value if and only if $\varphi>k$

(ii) a financing project creates value if and only if $\varphi<k$.

Magni $(2010,2013)$ showed that, if $\varphi=\bar{\imath}$, then the metric is NPV-consistent, since, for any $c$,

$$
\operatorname{NPV}(1+k)=C(\bar{\imath}-k) .
$$

The above definition and eq. (4) are particularly interesting because they show that the AIRR approach enables a deeper inspection of the economic content of the project than the traditional NPV analysis. Indeed, NPV is rewritten in terms of product of a capital base $C$ and an excess return $\bar{\imath}-k$. This means that the economic value created is determined by two factors: The project scale $(C)$ and the project $\bar{\imath}-k$. The same NPV can be created either by investing a large capital amount at a small rate or investing a small capital at a high rate. The AIRR approach enables decomposing value creation into important factors. Furthermore, the general definition stated above enables the analyst to understand whether value is created because capital is invested at a rate of return which is higher than the $\mathrm{COC}$ or because capital is borrowed at a financing rate which is smaller than the COC.

A shortcut for computing of AIRR is available from (4):

$$
\bar{\imath}(C)=k+\frac{\mathrm{NPV}}{C}(1+k)
$$

where dependence on the overall capital $C$ is highlighted.

The internal rate of return (IRR), here denoted as $x$, is the discount rate such that NPV is zero: $\operatorname{NPV}(x)=0$. Magni $(2010,2013)$ showed that IRR is a special case of AIRR obtained by assuming that weighted average of period rates is associated with a class of capital streams that are equivalent to the Hotelling values. Hotelling value, here denoted as $c_{t}(x)$, is such that the constant force of interest is constant:

$$
c_{t}(x)=c_{t-1}(x)(1+x)-F_{t}
$$

(see Magni 2010, 2013). This means that the capital is assumed to appreciate exponentially between two cash-flow dates and then decreases (or increases) by the distributed 
(or contributed) amount $F_{t} \cdot{ }^{1}$ Therefore, from (5),

$$
x=\bar{\imath}\left(C^{x}\right)=k+\frac{\mathrm{NPV}}{C^{x}}(1+k)
$$

where $C^{x}=\sum_{t=1}^{p} c_{t-1}(x)(1+k)^{-(t-1)}$.

We now consider the (somewhat opposite) assumption of capital that depreciates linearly with time: $c_{t}=c_{0}(1-\gamma t)$ where $\gamma=1 / p$. In essence, this means that a straight-line (SL) depreciation for capital is assumed: The associated AIRR is

$$
\bar{\imath}\left(C^{S L}\right)=k+\frac{\mathrm{NPV}}{C^{S L}}(1+k)
$$

where $C^{S L}=\sum_{t=1}^{p}\left(c_{0} \cdot\left(1-\frac{t-1}{p}\right)\right)(1+k)^{-(t-1)}$.

IRR and the straight-line AIRR (SL-AIRR) are two special cases of AIRR, associated with different classes of capital streams. As (4) holds no matter what the capital stream is, both IRR and SL-AIRR (both belonging to the AIRR class) are NPV-consistent (see also Hazen 2003 on the NPV-consistency of IRR).

However, note that IRR suffers from many well-known or lesser-known difficulties (see Magni 2013 for a compendium). For example, it may or may not exist or multiple IRRs may exist. Also, the financial nature of the IRR depends upon the COC, $k$, as the sign of $C$ is not necessarily invariant under changes in $k$. Contrary to IRR, the SL-AIRR has the nice property of existence and uniqueness for any project. Also, its financial nature is unambiguously determined by the sign of $c_{0}$, which coincides with the sign of $C$ for any given $k: C^{S L}>0$ if and only if $c_{0}>0$.

This makes SL-AIRR an interesting candidate as a reliable measure of worth, consistent with NPV and immune from the difficulties that mar the IRR.

Example 1. Consider a project $P$ such that $F=(-10,23,-17,24,-22)$ and a COC equal to $k=32 \%$. Two IRRs exist: $x(1)=11.2 \%$ and $x(2)=67 \%$. The former is associated with the Hotelling stream $c=(10,-6.3,6.5,-13.2,0)$, the latter is associated with the Hotelling stream $c=(10,-11.9,3.8,-19.8,0)$. The overall capital associated with $x(1)$ is $C^{x(1)}=2.4$, the overall capital associated with $x(2)$ is $C^{x(2)}=-4.1$. Therefore, IRR does not unambiguously determine the financial nature of the project: According to the first IRR, the project is an investment, according to the second IRR the project is a financing. Conversely, the SL-AIRR exists and is unique in any case, and unambiguously identifies the project as an investment, since the associated capital stream is $c=(10,7.5,5,2.5)$ so that the total capital invested is $C^{S L}=14.9>0$. The SL-AIRR is then $\bar{\imath}\left(C^{S L}\right)=$ $0.32+0.86(1+0.32) / 14.9=37.8 \%$. The investment is worth undertaking, given that $\bar{\imath}\left(C^{S L}\right)>k$.

Owing to (2), the NPV is a function of several value drivers: (i) The revenues $\left(R_{t}\right)$, (ii) the cost of goods sold, the selling, general and administrative costs (all included in $C_{t}$ ), (iii) the accounts receivable and payable, the inventory, the liquid assets (all included in $W C_{t}$ ), (iv) the depreciation charge for $N F A$ and the capital expenditures (included in $\left.N F A_{t}-N F A_{t-1}\right)^{2}$ (v) the tax rate $(\tau)$.

The IRR is an implicit function of the value drivers as well:

$$
\sum_{t=0}^{p}\left(\left(R_{t}-C_{t}-D e p_{t}\right)(1-\tau)-\left(N F A_{t}-N F A_{t-1}\right)-\left(W C_{t}-W C_{t-1}\right)\right)(1+x)^{-t}=0 .
$$

It is also evident that the SL-AIRR depends on value drivers as well, being a function of NPV.

\footnotetext{
${ }^{1}$ For example, the cash-flow stream $F=(-100,0,0,40,0,80)$ has a unique IRR equal to $x=4.32 \%$. The capital at time 3 is $100(1+0.0432)^{3}-40=73.52$.

${ }^{2} \mathrm{Dep}_{t}$ is included in both the income statement (it affects $I_{t}$ ) and the accruals (it affects $N F A_{t}-N F A_{t-1}$ )
} 
Our aim is to check whether the coherence of IRR, SL-AIRR and NPV, which is guaranteed in a traditional sense, remains valid if changes in value drivers are considered to take account of the uncertainty of the estimated value drivers. The analysis of change in a model's inputs and the impact on the model output is the purpose of Sensitivity Analysis (SA).

Figure 1 graphically describes the AIRR function $\bar{\imath}(C)$ for a value-creating project; among the infinitely many AIRRs, we highlight the SL-AIRR and one IRR.

From now on, we will only deal with SL-AIRR, so we will use the symbol $\bar{\imath}$ to denote it, omitting the dependence on $C^{S L}$ to avoid notational pedantry.

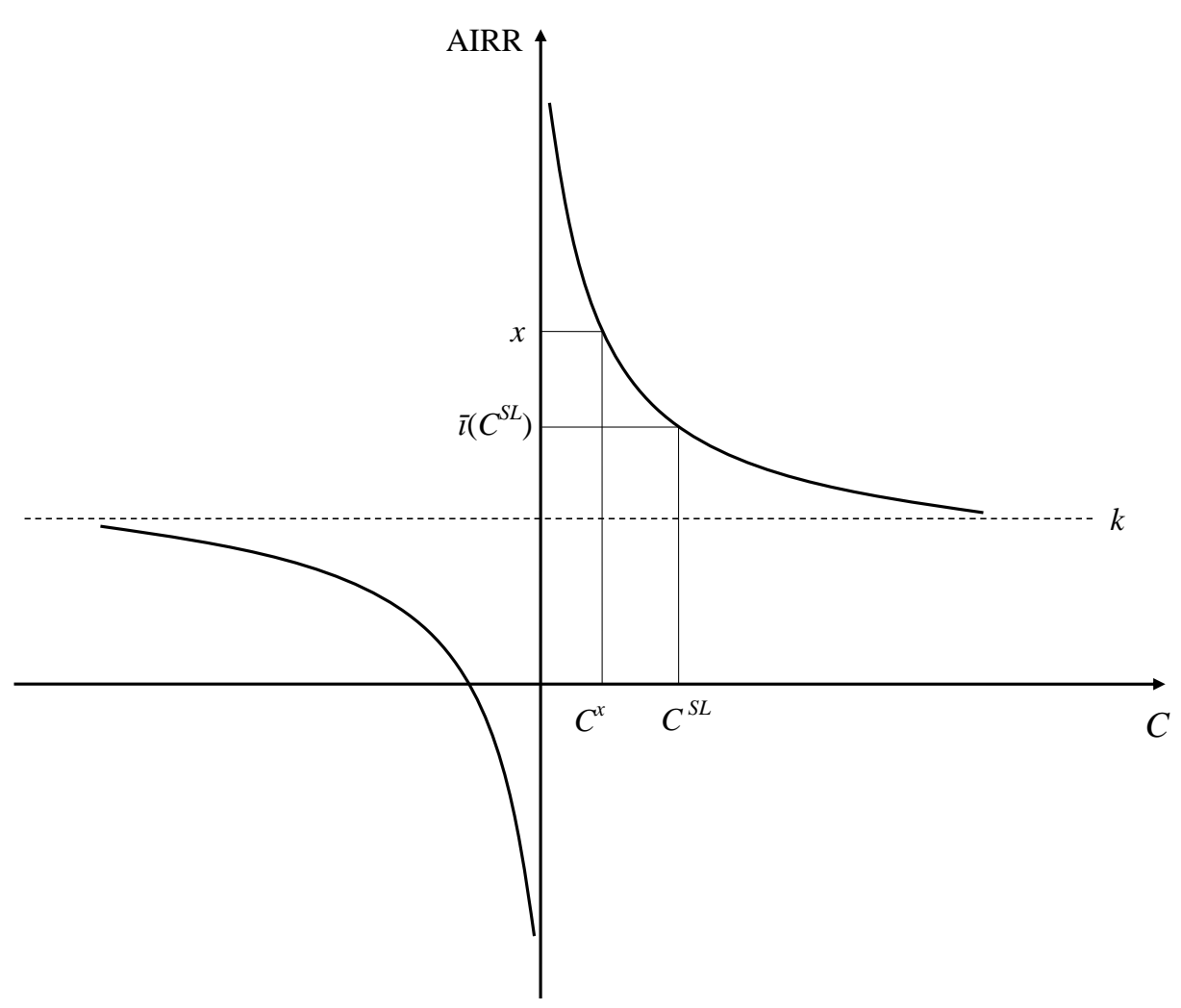

Figure 1: Graph of the AIRR function for a positive-NPV project

\section{Sensitivity analysis}

In the definition of Saltelli et al. (2004, p. 45), sensitivity analysis (SA) is the "study of how the uncertainty in the output of a model (numerical or otherwise) can be apportioned to different sources of uncertainty in the model input."

Given a model and a set of inputs (parameters), the SA investigates the relevance of parameters in terms of variability of the model output. In the literature there exist many SA techniques (see Pianosi et al. 2014, Borgonovo and Plischke 2016 for review of SA methods). The choice of an SA technique which best suits the model depends on several factors, among which the purpose of the analysis, the size of the variation of the parameters and the computational cost of the analysis.

A model can be described as consisting of an objective function $f$ defined on the 
parameter space $A$, which maps vector of inputs onto an output model $y$ :

$$
f: A \subset \mathbb{R}^{n} \rightarrow \mathbb{R}, \quad y=f(\alpha), \quad \alpha=\left(\alpha_{1}, \alpha_{2}, \ldots, \alpha_{n}\right) .
$$

The vector $\alpha=\left(\alpha_{1}, \alpha_{2}, \ldots, \alpha_{n}\right) \in A \subset \mathbb{R}^{n}$ is the vector of inputs or parameters or key drivers and $y(\alpha)$ is the output of the model. Let $\alpha^{0}=\left(\alpha_{1}^{0}, \alpha_{2}^{0}, \ldots, \alpha_{n}^{0}\right) \in A$ be the basecase, a representative value (e.g., mean value, most probable value, etc.). The relevance of a parameter $\alpha_{i}$ (also known as importance measure) quantifies the impact of $\alpha_{i}$ on the output variation. Let $R^{f}=\left(R_{1}^{f}, R_{2}^{f}, \ldots, R_{n}^{f}\right)$ be the vector of the relevances. The latter determines the ranking of the parameters in the following way. Input $\alpha_{i}$ is defined to be more relevant than $\alpha_{j}$ if and only $\left|R_{i}^{f}\right|>\left|R_{j}^{f}\right|$. The parameters are equally relevant for $f$ if $\left|R_{i}^{f}\right|=\left|R_{j}^{f}\right|$. The rank of $\alpha_{i}$, denoted as $r_{i}^{f}$, depends on the importance measure: $\alpha_{i}$ has a higher rank (it has a greater impact on the output) than $\alpha_{j}$ if it has greater relevance. Let $r^{f}=\left(r_{1}^{f}, r_{2}^{f}, \ldots, r_{n}^{f}\right)$ be the vector of ranks.

The average rank is $r_{M}^{f}=\frac{\sum_{i=1}^{n} i}{n}=\frac{\frac{n \cdot(n+1)}{2}}{n}=\frac{n+1}{2}$.

The high parameters (or high-relevance parameters) are those whose rank is higher than the average rank $r_{M}^{f}$; the low parameters are those parameters whose rank is smaller than $r_{M}^{f}$.

Example 2. Consider $A=\left\{\alpha_{1}, \alpha_{2}, \alpha_{3}, \alpha_{4}, \alpha_{5}\right\}$ and the two objective functions $f$ and $g$. Given an SA technique, suppose the vector of importance measures for $f$ is $R^{f}=$ $(0.1,0.3,0.2,0.05,0.35)$. No relevance is equal (i.e., there are no ties) so the rank vector is $r^{f}=(4,2,3,5,1)$. Suppose the vector of importance measures for $g$ is $R^{g}=$ $(0.05,0.4,0.2,0.2,0.15)$. Two relevances are equal (there are ties). Input $\alpha_{3}$ and $\alpha_{4}$ are equally ranked. In this case, the inputted rank is $\frac{2+3}{2}=2.5$ for both. The rank vector is then $r^{g}=(5,1,2.5,2.5,4)$.

In the literature, SA techniques can be divided into global SA techniques and local SA techniques.

Global SA measures the importance of key parameters within the entire parameter space $A$. Local SA measures the importance of parameters in a neighborhood of $\alpha^{0}$ and makes use of Taylor approximation. This approach presupposes that the objective function is differentiable in $\alpha^{0}$ and the changes in the inputs are small.

Following we briefly describe some well-known SA techniques.

(i) Standardized regression coefficient (global SA)

Let $V$ denote variance and $\sigma$ denote standard deviation. Consider the linear regression with dependent variable $f$ and explanatory variables $\alpha_{i}, \forall i=1, \ldots, n$, estimated with OLS method: $f=\beta_{0}^{f}+\sum_{i=1}^{n} \beta_{i}^{f} \cdot \alpha_{i}+u$. The standardized regression coefficient $S R C_{i}^{f}$ measures the importance of $\alpha_{i}$ (Saltelli and Marivoet 1990, Bring 1994, Saltelli et al. 2008):

$$
S R C_{i}^{f}=\frac{\beta_{i}^{f} \cdot \sigma\left(\alpha_{i}\right)}{\sigma(f)} .
$$

(ii) Sensitivity indices in variance-based decomposition methods (global SA)

Variance-based methods study how the variance of the output is affected by (and apportioned to) the uncertain input factors. In variance-based methods, the importance of a parameter is generally represented through the First Order Sensitivity Index (FOSI) and the Total Order Sensitivity Index (TOSI) (Saltelli et al. 2008). The FOSI, here denoted as $S I_{i}^{1, f}$, measures the individual effect of the parameter on the output variance:

$$
S I_{i}^{1, f}=\frac{V\left(E\left(f \mid \alpha_{i}\right)\right)}{V(f)}
$$


where $V\left(E\left(f \mid \alpha_{i}\right)\right)$ is the variance of the expectation of $f$ upon a fixed value of $\alpha_{i}{ }^{3}$ The TOSI, here denoted as $S I_{i}^{T, f}$, measures the total contribution of $\alpha_{i}$ to the output variability, i.e., it is inclusive of the interaction effects with other parameters or groups of parameters. $S I_{i}^{T, f}$ can be calculated as (Saltelli et al. 2008)

$$
S I_{i}^{T, f}=\frac{E\left(V\left(f \mid \alpha_{-i}\right)\right)}{V(f)},
$$

where $f\left|\alpha_{-i}=f\right| \alpha_{1}, \alpha_{2}, \ldots, \alpha_{i-1}, \alpha_{i+1}, \ldots, \alpha_{n}{ }^{4}$

\section{(iii) Finite Change Sensitivity Indices (global SA)}

The Finite Change Sensitivity Indices (FCSIs), introduced in Borgonovo (2010a, 2010b), focus on the output change due to a finite input change; they are based on the properties of functional ANOVA decomposition for finite changes (Rabitz and Alis 1999, Borgonovo 2010a). There exist two versions of FCSIs: First Order FCSI and Total Order FCSI.

The First Order FCSI of a parameter measures the individual effect of the parameter's variation on $f$; the Total Order FCSI considers the total effect of a parameter's variation on $f$, including both the individual contribution and the interactions between a parameter and the other parameters.

Consider the base value $\alpha^{0}=\left(\alpha_{1}^{0}, \ldots, \alpha_{n}^{0}\right)$; the corresponding output is $f\left(\alpha^{0}\right)$. The parameters change from $\alpha^{0}$ to $\alpha^{1}=\left(\alpha_{1}^{1}, \alpha_{2}^{1}, \ldots, \alpha_{n}^{1}\right) \in A$ and the corresponding output is $f\left(\alpha^{1}\right)$. The output variation is $\Delta f=f\left(\alpha^{1}\right)-f\left(\alpha^{0}\right)$.

Let $\left(\alpha_{i}^{1}, \alpha_{(-i)}^{0}\right)=\left(\alpha_{1}^{0}, \alpha_{2}^{0}, \ldots, \alpha_{i-1}^{0}, \alpha_{i}^{1}, \alpha_{i+1}^{0}, \ldots, \alpha_{n}^{0}\right)$ be obtained by varying the parameter $\alpha_{i}$ to the new value $\alpha_{i}^{1}$, while the remaining $n-1$ parameters are fixed at $\alpha^{0}$. Similarly, $\left(\alpha_{i}^{1}, \alpha_{j}^{1}, \alpha_{(-i, j)}^{0}\right)=\left(\alpha_{1}^{0}, \alpha_{2}^{0}, \ldots, \alpha_{i-1}^{0}, \alpha_{i}^{1}, \alpha_{i+1}^{0}, \ldots, \alpha_{j-1}^{0}, \alpha_{j}^{1}, \alpha_{j+1}^{0}, \ldots, \alpha_{n}^{0}\right)$ is the vector of inputs assuming $\alpha_{i}$ and $\alpha_{j}$ are set to the new values, while the remaining $n-2$ are unvaried, and so forth for all $j$-tuples of inputs, $j=1,2, \ldots, n$.

The individual effect of $\alpha_{i}$ on $\Delta f$ is $\Delta_{i} f=f\left(\alpha_{i}^{1}, \alpha_{(-i)}^{0}\right)-f\left(\alpha^{0}\right)$ and the First Order FCSI of $\alpha_{i}$, denoted as $\Phi_{i}^{1, f}$, is (Borgonovo 2010a):

$$
\Phi_{i}^{1, f}=\frac{\Delta_{i} f}{\Delta f} .
$$

The interaction between $\alpha_{i}$ and $\alpha_{j}, \Delta_{i, j} f$, is the portion of $f\left(\alpha_{i}^{1}, \alpha_{j}^{1}, \alpha_{(-i, j)}^{0}\right)-f\left(\alpha^{0}\right)$ that is not explained by the individual effects $\Delta_{i} f$ and $\Delta_{j} f: \Delta_{i, j} f=f\left(\alpha_{i}^{1}, \alpha_{j}^{1}, \alpha_{(-i, j)}^{0}\right)-$ $f\left(\alpha^{0}\right)-\Delta_{i} f-\Delta_{j} f$. Likewise, the interaction between the triplet of inputs $\alpha_{i}, \alpha_{j}$ and $\alpha_{w}$, denoted as $\Delta_{i, j, w} f$, is the portion of $f\left(\alpha_{i}^{1}, \alpha_{j}^{1}, \alpha_{w}^{1}, \alpha_{(-i, j, w)}^{0}\right)-f\left(\alpha^{0}\right)$ that is not explained by the individual effects and the interactions between all the possible pairs of inputs $\alpha_{i}$, $\alpha_{j}$ and $\alpha_{w}$ :

$$
\Delta_{i, j, w} f=f\left(\alpha_{i}^{1}, \alpha_{j}^{1}, \alpha_{w}^{1}, \alpha_{(-i, j, w)}^{0}\right)-f\left(\alpha^{0}\right)-\Delta_{i} f-\Delta_{j} f-\Delta_{w} f-\Delta_{i, j} f-\Delta_{i, w} f-\Delta_{j, w} f
$$

(analogously for a group of $s>3$ parameters).

\footnotetext{
${ }^{3}$ It can be shown that $V\left(E\left(f \mid \alpha_{i}\right)\right)=V(f)-E\left[V\left(f \mid \alpha_{i}\right)\right]$ (see Satelli et al. 2008).

${ }^{4}$ Among the various variance-based decomposition methods, the High Dimensional Model Representation (HDMR) theory allows a complete decomposition of the output variance through a finite number of terms, under the assumption of independence (i.e., orthogonality) of the input factors (Sobol' 1993, Sobol' 2001, Saltelli et al. 2008):

$$
V(f)=\sum_{i=1}^{n} V_{i}^{f}+\sum_{i<j} V_{i, j}^{f}+\sum_{i<j<m} V_{i, j, m}^{f}+\cdots+V_{1,2, \ldots, n}^{f},
$$

where $V_{i}^{f}=V\left(E\left(f \mid \alpha_{i}\right)\right) ; V_{i, j}^{f}$ is the interaction between $\alpha_{i}$ and $\alpha_{j} ; V_{i, j, m}^{f}$ is the interaction among $\alpha_{i}, \alpha_{j}$ and $\alpha_{m}$ and, finally, $V_{1,2, \ldots, n}^{f}$ is the residual portion of variance, explained by the interaction among all the $n$ parameters.
} 
$\Delta f$ is equal to the sum of individual effects and interactions between parameters and groups of parameters (Borgonovo 2010a):

$$
\Delta f=\sum_{i=1}^{n} \Delta_{i} f+\sum_{s=2}^{n} \sum_{i_{1}<i_{2} \cdots<i_{s}} \Delta_{i_{1}, i_{2}, \ldots, i_{s}} f,
$$

where $\sum_{i_{1}<i_{2} \cdots<i_{s}} \Delta_{i_{1}, i_{2}, \ldots, i_{s}} f$ is the sum of the interactions between groups of $s$ parameters.

The total effect of the parameter $\alpha_{i}$, denoted as $\Delta_{i}^{T} f$, is the sum of the individual effect of $\alpha_{i}$ and of the interactions that involve $\alpha_{i}$ :

$$
\Delta_{i}^{T} f=\Delta_{i} f+\sum_{s=2}^{n} \sum_{\substack{i_{1}<i_{2} \ldots<i_{s} \\ i \in\left\{i_{1}, i_{2}, \ldots, i_{s}\right\}}} \Delta_{i_{1}, i_{2}, \ldots, i_{s}} f .
$$

Borgonovo (2010a, Proposition 1) showed that $\Delta_{i}^{T} f$ is also obtained as

$$
\Delta_{i}^{T} f=f\left(\alpha^{1}\right)-f\left(\alpha_{i}^{0}, \alpha_{(-i)}^{1}\right), \forall i=1,2, \ldots, n,
$$

where $\left(\alpha_{i}^{0}, \alpha_{(-i)}^{1}\right)$ is the point with all the parameters equal to the new value $\alpha^{1}$, except the parameter $\alpha_{i}$, which is equal to $\alpha_{i}^{0}$. The Total Order FCSI of the parameter $\alpha_{i}$, denoted as $\Phi_{i}^{T, f}$, is (Borgonovo 2010a):

$$
\Phi_{i}^{T, f}=\frac{\Delta_{i}^{T} f}{\Delta f}=\frac{f\left(\alpha^{1}\right)-f\left(\alpha_{i}^{0}, \alpha_{(-i)}^{1}\right)}{\Delta f} .
$$

(iv) Helton's index (local SA)

Helton (1993) proposed a variance decomposition of $f$ based on Taylor approximation. He assumed parameters are not correlated, so the variance of $f$ can be approximated by

$$
\hat{V}(f)=\sum_{i=1}^{n}\left[f_{\alpha_{i}}^{\prime}\left(\alpha^{0}\right)\right]^{2} \cdot V\left(\alpha_{i}\right) .
$$

The impact of input $\alpha_{i}$ on $V(f)$ can be measured by

$$
H_{i}^{f}\left(\alpha^{0}\right)=\frac{\left[f_{\alpha_{i}}^{\prime}\left(\alpha^{0}\right)\right]^{2} \cdot V\left(\alpha_{i}\right)}{\hat{V}(f)} .
$$

\section{(v) Normalized Partial Derivatives (local SA)}

Helton (1993) also proposed the adoption of normalized partial derivatives as sensitivity measures. He defined two versions of normalized partial derivatives (NPDs):

$$
\begin{aligned}
& N P D 1_{i}^{f}\left(\alpha^{0}\right)=f_{\alpha_{i}}^{\prime}\left(\alpha^{0}\right) \cdot \frac{\alpha_{i}^{0}}{f\left(\alpha^{0}\right)}, \\
& N P D 2_{i}^{f}\left(\alpha^{0}\right)=f_{\alpha_{i}}^{\prime}\left(\alpha^{0}\right) \cdot \frac{\sigma\left(\alpha_{i}\right)}{\hat{\sigma}(f)},
\end{aligned}
$$

where $\hat{\sigma}(f)$ is the square root of $\hat{V}(f)$ defined in $(14)$. NPD ${ }_{i}^{f}\left(\alpha^{0}\right)$ measures the elasticity of $f$ with respect to $\alpha$ in $\alpha^{0}$ assuming that the relative change in $\alpha_{i}$ is fixed for $i=$ $1,2, \ldots, n$ (Helton 1993, p. 329). NPD2 $2_{i}^{f}\left(\alpha^{0}\right)$ is the square root of (15).

(vi) Differential Importance Measure (local SA)

The total variation $f\left(\alpha^{0}+\mathrm{d} \alpha\right)-f\left(\alpha^{0}\right)$ of a differentiable function $f$ due to a local change $\mathrm{d} \alpha$ can be approximated by the total differential

$$
\mathrm{d} f=\sum_{i=1}^{n} f_{\alpha_{i}}^{\prime}\left(\alpha^{0}\right) \cdot \mathrm{d} \alpha_{i} .
$$


The Differential Importance Measure (DIM) of parameter $\alpha_{i}$ is the ratio of the partial differential of $f$ with respect to $\alpha_{i}$ to the total differential of $f$ (Borgonovo and Apostolakis 2001, Borgonovo and Peccati 2004):

$$
D I M_{i}^{f}\left(\alpha^{0}, \mathrm{~d} \alpha\right)=\frac{\mathrm{d} f_{a_{i}}}{\mathrm{~d} f}=\frac{f_{\alpha_{i}}^{\prime}\left(\alpha^{0}\right) \cdot \mathrm{d} \alpha_{i}}{\sum_{j=1}^{n} f_{\alpha_{j}}^{\prime}\left(\alpha^{0}\right) \cdot \mathrm{d} \alpha_{j}} .
$$

The DIM of a parameter represents the percentage of the function's variation due to the variation of that parameter (Borgonovo and Apostolakis 2001, Borgonovo and Peccati 2004).

There are two versions of DIM, according to the assumption made upon the variation structure of parameters: Uniform variation assumption (H1) or proportional variation assumption (H2). $\mathrm{H} 1$ implies $\mathrm{d} \alpha_{i}=\mathrm{d} \alpha_{j}, \forall \alpha_{i}, \alpha_{j}$. This assumption can be validly accepted only if all the parameters are expressed in the same unit of measure. The resulting DIM is

$$
D I M 1_{i}^{f}\left(\alpha^{0}\right)=\frac{f_{\alpha_{i}}^{\prime}\left(\alpha^{0}\right) \cdot \mathrm{d} \alpha_{i}}{\sum_{j=1}^{n} f_{\alpha_{j}}^{\prime}\left(\alpha^{0}\right) \cdot \mathrm{d} \alpha_{i}}=\frac{f_{\alpha_{i}}^{\prime}\left(\alpha^{0}\right)}{\sum_{j=1}^{n} f_{\alpha_{j}}^{\prime}\left(\alpha^{0}\right)} .
$$

H2 implies $\mathrm{d} \alpha_{i}=\xi \cdot \alpha_{i}^{0}$ for some $\xi \neq 0$. This assumption can be adopted even when the parameters are expressed in different units of measure, because the parameters' variation is defined with respect to the base value of each parameter (Borgonovo and Apostolakis 2001, Borgonovo and Peccati 2004). The resulting DIM is

$$
D I M 2_{i}^{f}\left(\alpha^{0}\right)=\frac{f_{\alpha_{i}}^{\prime}\left(\alpha^{0}\right) \cdot \xi \cdot \alpha_{i}^{0}}{\sum_{j=1}^{n} f_{\alpha_{j}}^{\prime}\left(\alpha^{0}\right) \cdot \xi \cdot \alpha_{j}^{0}}=\frac{f_{\alpha_{i}}^{\prime}\left(\alpha^{0}\right) \cdot \alpha_{i}^{0}}{\sum_{j=1}^{n} f_{\alpha_{j}}^{\prime}\left(\alpha^{0}\right) \cdot \alpha_{j}^{0}} .
$$

\section{Coherence between objective functions}

Risk management problems are often characterized by the definition of more than one objective function (Borgonovo and Peccati 2006, Borgonovo, Gatti and Peccati 2010). For a given technique, the analysis can be applied using different objective functions. A relevant aspect is the evaluation of the coherence (or compatibility) between the results of the sensitivity analysis for different functions.

We consider the objective functions $f, g: A \rightarrow \mathbb{R}$. The vector of importance measures are respectively $R^{f}=\left(R_{1}^{f}, R_{2}^{f}, \ldots, R_{n}^{f}\right)$ and $R^{g}=\left(R_{1}^{g}, R_{2}^{g}, \ldots, R_{n}^{g}\right)$; the ranking vectors are $r^{f}=\left(r_{1}^{f}, r_{2}^{f}, \ldots, r_{n}^{f}\right)$ and $r^{g}=\left(r_{1}^{g}, r_{2}^{g}, \ldots, r_{n}^{g}\right)$.

Definition 3. (Coherence) Given a technique of $S A$ and two objective functions $f$ and $g$, they are coherent if the ranking vectors coincide: $r^{f}=r^{g}$. If, in addition, the vectors of the relevances coincide, $R^{f}=R^{g}$, they are strictly coherent.

Example 3. Consider the functions $f$ and $g$ and assume $R^{f}=(0.1,-0.3,0.2,0.05,0.35)$ and $R^{g}=(0.07,0.35,0.15,0.03,0.40)$. Recalling that the rank is determined by the absolute value of the importance measure, the functions $f$ and $g$ determine the same ranking, $r^{f}=r^{g}=(4,2,3,5,1)$, so $f$ and $g$ are coherent but not strictly coherent.

If two functions $f$ and $g$ are not coherent, the degree of incoherence can be alternatively measured through Spearman's rank correlation coefficient (Spearman 1904) or top-down correlation coefficient (Iman and Conover 1987).

Spearman's rank correlation coefficient (henceforth, Spearman's coefficient) between two stochastic variables is the correlation coefficient between the ranks of the stochastic variables (Spearman 1904). In SA, Spearman's coefficient between two objective functions $f$ and $g$, denoted as $\rho_{f, g}$, is the correlation coefficient of the ranking vectors $r^{f}$ and $r^{g}$ :

$$
\rho_{f, g}=\frac{\operatorname{Cov}\left(r^{f}, r^{g}\right)}{\sigma\left(r^{f}\right) \cdot \sigma\left(r^{g}\right)}=\frac{\sum_{i=1}^{n}\left(r_{i}^{f}-r_{M}^{f}\right) \cdot\left(r_{i}^{g}-r_{M}^{g}\right)}{\sqrt{\sum_{i=1}^{n}\left(r_{i}^{f}-r_{M}^{f}\right)^{2}} \cdot \sqrt{\sum_{i=1}^{n}\left(r_{i}^{g}-r_{M}^{g}\right)^{2}}},
$$


where, as seen, $r_{M}^{f}=r_{M}^{g}=\frac{n+1}{2}$. The coefficient $\rho_{f, g}$ attributes the same weight to top and low parameters and lies in the interval $[-1,1]$. The coefficient $\rho_{f, g}$ is equal to 1 if and only if $f$ and $g$ are coherent according to Definition 3. Therefore, a value of $\rho_{f, g}$ smaller than 1 signals incoherence between $f$ and $g$ : The smaller the value of $\rho_{f, g}$, the higher the degree of incoherence. The difference $1-\rho_{f, g}$ can be taken as representative of the degree of incoherence. Iman and Conover (1987) introduced the top-down correlation coefficient, a compatibility measure that attributes a higher weight to top parameters than to low parameters. This measure is based on Savage Score (Savage 1956). The Savage score of parameter $\alpha_{i}$, denoted as $S_{i}^{f}$, is

$$
S_{i}^{f}=\sum_{h=r_{i}^{f}}^{n} \frac{1}{h}
$$

$S^{f}=\left(S_{1}^{f}, S_{2}^{f}, \ldots, S_{n}^{f}\right)$ is the Savage scores' vector. The average Savage score is $S_{M}^{f}=$ $\frac{\sum_{i=1}^{n} S_{i}^{f}}{n}=1$. Table 1 shows the value of Savage scores for $n=5$.

Table 1: Savage scores for $n=5$

\begin{tabular}{ll}
\hline$r_{i}^{f} \quad S_{i}^{f}$ \\
\hline $1 \quad \sum_{h=1}^{5} \frac{1}{h}=\frac{1}{1}+\frac{1}{2}+\frac{1}{3}+\frac{1}{4}+\frac{1}{5}=2.28 \overline{3}$ \\
$2 \quad \sum_{h=2}^{5} \frac{1}{h}=\frac{1}{2}+\frac{1}{3}+\frac{1}{4}+\frac{1}{5}=1.28 \overline{3}$ \\
$3 \quad \sum_{h=3}^{5} \frac{1}{h}=\frac{1}{3}+\frac{1}{4}+\frac{1}{5}=0.78 \overline{3}$ \\
$4 \quad \sum_{h=4}^{5} \frac{1}{h}=\frac{1}{4}+\frac{1}{5}=0.45$ \\
$5 \quad \sum_{h=5}^{5} \frac{1}{h}=\frac{1}{5}=0.2$ \\
\hline
\end{tabular}

The top-down correlation coefficient between the objective functions $f$ and $g$, denoted as $\rho_{S^{f}, S^{g}}$, is the correlation coefficient between the Savage scores' vectors $S^{f}$ and $S^{g}$ (Iman and Conover 1987):

$$
\rho_{S^{f}, S^{g}}=\frac{\operatorname{Cov}\left(S^{f}, S^{g}\right)}{\sigma\left(S^{f}\right) \cdot \sigma\left(S^{g}\right)}=\frac{\sum_{i=1}^{n}\left(S_{i}^{f}-S_{M}^{f}\right) \cdot\left(S_{i}^{g}-S_{M}^{g}\right)}{\sqrt{\sum_{i=1}^{n}\left(S_{i}^{f}-S_{M}^{f}\right)^{2}} \cdot \sqrt{\sum_{i=1}^{n}\left(S_{i}^{g}-S_{M}^{g}\right)^{2}}},
$$

where $S_{M}^{f}=S_{M}^{g}=1$. The coefficient $\rho_{S^{f}, S^{g}}$ measures the compatibility between the parameters' ranking of $f$ and $g$ : The accordance between top parameters determines a remarkable influence on $\rho_{S^{f}, S^{g}}$, while the discordance between low parameters has a weak influence on $\rho_{S^{f}, S^{g}}$ (Iman and Conover 1987).

If the aim of the analysis is factor prioritization (i.e., identification of the most relevant parameters), the top-down coefficient should be preferred to Spearman's coefficient.

The maximum value of $\rho_{S^{f}, S^{g}}$ is equal to 1 . In case $f$ and $g$ have no ties, the minimum value is -1 for $n=2$, it increases as $n$ increases, and it tends to -0.645 as $n$ tend to infinity (Iman and Conover 1987).

$\rho_{S^{f}, S^{g}}$ is equal to 1 if and only if $f$ and $g$ are strictly coherent. Therefore, a value of $\rho_{S^{f}, S^{g}}$ smaller than 1 signals incompatibility between $f$ and $g$. The smaller the value of 
$\rho_{S^{f}, S^{g}}$, the higher the incoherence level. The degree of incoherence of $f$ and $g$ can then be measured by $1-\rho_{S^{f}, S^{g}}$.

Borgonovo et al. (2014) showed that an objective function $f$ and a monotonic transformation $g$ of it generate the same ranking of the parameters under several techniques. This means that they are coherent according to Definition 3.

We now show that, if $g$ is an affine transformation of $f$, that is, $g(\alpha)=l \cdot f(\alpha)+q$ for all $\alpha \in A$, then $f$ and $g$ are strictly coherent under several techniques.

Proposition 1. A function and an affine transformation of it are strictly coherent under the following techniques:

(i) Standardized regression coefficient

(ii) Sensitivity Indices in variance-based decomposition models

(iii) Finite Change Sensitivity Indices

(iv) Helton's index

(v) Normalized Partial Derivative (NPD2)

(vi) Differential Importance Measure.

Proof. By hypothesis, $g(\alpha)=l \cdot f(\alpha)+q$. Therefore,

(i) $g=l \cdot\left(\beta_{0}^{f}+\sum_{i=1}^{n} \beta_{i}^{f} \cdot \alpha_{i}+u\right)+q=\left(l \cdot \beta_{0}^{f}+q\right)+\sum_{i=1}^{n}\left(l \cdot \beta_{i}^{f}\right) \cdot \alpha_{i}+l \cdot u$, whence

$$
\begin{aligned}
& \beta_{0}^{g}=l \cdot \beta_{0}^{f}+q, \\
& \beta_{i}^{g}=l \cdot \beta_{i}^{f}
\end{aligned}
$$

so that

$$
S R C_{i}^{g}=\frac{\beta_{i}^{g} \cdot \sigma\left(\alpha_{i}\right)}{\sigma(g)}=\frac{l \cdot \beta_{i}^{f} \cdot \sigma\left(\alpha_{i}\right)}{l \cdot \sigma(f)}=S R C_{i}^{f} .
$$

(ii) Denoting as $f \mid \alpha_{i}$ (and $g \mid \alpha_{i}$ ) the function $f$ (and $g$ ) conditional to a specific value of $\alpha_{i}, g\left|\alpha_{i}=(l \cdot f+q)\right| \alpha_{i}=(l \cdot f)\left|\alpha_{i}+q=l \cdot f\right| \alpha_{i}+q$. Therefore,

$$
S I_{i}^{1, g}=\frac{V\left(E\left(g \mid \alpha_{i}\right)\right)}{V(g)}=\frac{V\left(E\left(l \cdot f \mid \alpha_{i}+q\right)\right)}{V(l \cdot f+q)}=\frac{l^{2} \cdot V\left(E\left(f \mid \alpha_{i}\right)\right)}{l^{2} \cdot V(f)}=S I_{i}^{1, f} .
$$

Analogously, $g\left|\alpha_{-i}=(l \cdot f+q)\right| \alpha_{-i}=l \cdot f \mid \alpha_{-i}+q$. Hence,

$$
S I_{i}^{T, g}=\frac{E\left(V\left(g \mid \alpha_{-i}\right)\right)}{V(f)}=\frac{E\left(V\left(l \cdot f \mid \alpha_{-i}+q\right)\right)}{V(l \cdot f+q)}=\frac{l^{2} \cdot E\left(V\left(f \mid \alpha_{-i}\right)\right)}{l^{2} \cdot V(f)}=S I_{i}^{T, f} .
$$

(iii) Since $\Delta g=g\left(\alpha^{1}\right)-g\left(\alpha^{0}\right)=l \cdot f\left(\alpha^{1}\right)+q-l \cdot f\left(\alpha^{0}\right)-q=l \cdot\left(f\left(\alpha^{1}\right)-f\left(\alpha_{0}\right)\right)=l \cdot \Delta f$, and

$$
\begin{aligned}
\Delta_{i} g & =g\left(\alpha_{i}^{1}, \alpha_{(-i)}^{0}\right)-g\left(\alpha^{0}\right)=l \cdot f\left(\alpha_{i}^{1}, \alpha_{(-i)}^{0}\right)+q-l \cdot f\left(\alpha^{0}\right)-q \\
& =l \cdot\left(f\left(\alpha_{i}^{1}, \alpha_{(-i)}^{0}\right)-f\left(\alpha^{0}\right)\right)=l \cdot \Delta_{i} f
\end{aligned}
$$

then

$$
\Phi_{i}^{1, g}=\frac{\Delta_{i} g}{\Delta g}=\frac{l \cdot \Delta_{i} f}{l \cdot \Delta f}=\frac{\Delta_{i} f}{\Delta f}=\Phi_{i}^{1, f} .
$$

As for the Total Indices

$$
\begin{aligned}
\Delta_{i}^{T} g & =g\left(\alpha^{1}\right)-g\left(\alpha_{i}^{0}, \alpha_{(-i)}^{1}\right)=l \cdot f\left(\alpha^{1}\right)+q-l \cdot f\left(\alpha_{i}^{0}, \alpha_{(-i)}^{1}\right)-q \\
& =l \cdot\left(f\left(\alpha^{1}\right)-f\left(\alpha_{i}^{0}, \alpha_{(-i)}^{1}\right)\right)=l \cdot \Delta_{i}^{T} f
\end{aligned}
$$

so that

$$
\Phi_{i}^{T, g}=\frac{\Delta_{i}^{T} g}{\Delta g}=\frac{l \cdot \Delta_{i}^{T} f}{l \cdot \Delta f}=\frac{\Delta_{i}^{T} f}{\Delta f}=\Phi_{i}^{T, f} .
$$


(iv) From (14),

$$
\begin{aligned}
\hat{V}(g) & =\sum_{i=1}^{n}\left[g_{\alpha_{i}}^{\prime}\left(\alpha^{0}\right)\right]^{2} \cdot V\left(\alpha_{i}\right) \\
& =\sum_{i=1}^{n}\left[l \cdot f_{\alpha_{i}}^{\prime}\left(\alpha^{0}\right)\right]^{2} \cdot V\left(\alpha_{i}\right)=l^{2} \cdot \sum_{i=1}^{n}\left[f_{\alpha_{i}}^{\prime}\left(\alpha^{0}\right)\right]^{2} \cdot V\left(\alpha_{i}\right)=l^{2} \cdot \hat{V}(f) .
\end{aligned}
$$

Hence,

$$
H_{i}^{g}\left(\alpha^{0}\right)=\frac{\left[g_{\alpha_{i}}^{\prime}\left(\alpha^{0}\right)\right]^{2} \cdot V\left(\alpha_{i}\right)}{\hat{V}(g)}=\frac{l^{2} \cdot\left[f_{\alpha_{i}}^{\prime}\left(\alpha^{0}\right)\right]^{2} \cdot V\left(\alpha_{i}\right)}{l^{2} \cdot \hat{V}(f)}=H_{i}^{f}\left(\alpha^{0}\right) .
$$

(v) Straightforward, since $N P D 2_{i}^{f}$ is the square root of $H_{i}^{f}\left(\alpha^{0}\right) .^{5}$

(vi) From (18),

$$
\begin{aligned}
D I M_{i}^{g}\left(\alpha^{0}, \mathrm{~d} \alpha\right) & =\frac{g_{\alpha_{i}}^{\prime}\left(\alpha^{0}\right) \cdot \mathrm{d} \alpha_{i}}{\sum_{j=1}^{n} g_{\alpha_{j}}^{\prime}\left(\alpha^{0}\right) \cdot \mathrm{d} \alpha_{j}}=\frac{l \cdot f_{\alpha_{i}}^{\prime}\left(\alpha^{0}\right) \cdot \mathrm{d} \alpha_{i}}{\sum_{j=1}^{n} l \cdot f_{\alpha_{j}}^{\prime}\left(\alpha^{0}\right) \cdot \mathrm{d} \alpha_{j}}=\frac{f_{\alpha_{i}}^{\prime}\left(\alpha^{0}\right) \cdot \mathrm{d} \alpha_{i}}{\sum_{j=1}^{n} f_{\alpha_{j}}^{\prime}\left(\alpha^{0}\right) \cdot \mathrm{d} \alpha_{j}} \\
& =D I M_{i}^{f}\left(\alpha^{0}, \mathrm{~d} \alpha\right) .
\end{aligned}
$$

(This result is independent of the structure of $\mathrm{d} \alpha$.)

Remark 1. While we have proved that, for several SA techniques, a function and its affine transformation are coherent (even in a strict sense), it is intuitive to inductively believe that a function and its affine transformation share an absolute coherence, in that they are coherent for every existing SA technique. We leave the proof of this more general statement for future research.

\section{Coherence between return rates and NPV}

The investment risk can be defined as "the potential variability of financial outcomes" (White et al. 1997). The future outcomes of an investment are stochastic and the investor has limited information. Referring to NPV and IRR, Joy and Bradley (1973, p. 1255) wrote: "It has often been suggested that capital budgeting theory has over-emphasized the development of such techniques with little regard for the typically poor data used in project evaluation and the effect that errors in capital budgeting inputs have on project profitability." The practise of valuation criteria should be corroborated by a careful investment risk analysis.

Given an investment model based on a set of value drivers, SA allows the evaluator to identify the most relevant parameters in terms of variation of the value. The most relevant parameters are the risk factors that mainly influence the investment. After SA has been performed, the investment risk can be reduced through information insights on the main risk factors identified by the analysis; the collection of extra information on these parameters allows more precise estimates and a remarkable uncertainty reduction

${ }^{5}$ It is worth noting that $f$ and $g$ are coherent but not strictly coherent under $N P D 2_{i}^{f}$ technique:

$$
N P D 1_{i}^{g}\left(\alpha^{0}\right)=g_{\alpha_{i}}^{\prime}\left(\alpha^{0}\right) \cdot \frac{\alpha_{i}^{0}}{g\left(\alpha^{0}\right)}=l \cdot f_{\alpha_{i}}^{\prime}\left(\alpha^{0}\right) \cdot \frac{\alpha_{i}^{0}}{g\left(\alpha^{0}\right)} \cdot \frac{f\left(\alpha^{0}\right)}{f\left(\alpha^{0}\right)}=l \cdot \frac{f\left(\alpha^{0}\right)}{g\left(\alpha^{0}\right)} \cdot N P D 1_{i}^{f}\left(\alpha^{0}\right)
$$

so that $\left|N P D 1_{i}^{f}\right|>\left|N P D 1_{j}^{f}\right|$ implies $\left|N P D 1_{i}^{g}\right|>\left|N P D 1_{j}^{g}\right|$. Therefore, the parameters' ranking in $f$ and $g$ is equal: $r^{f}=r^{g}$. 
(Borgonovo and Peccati 2006). Furthermore, the potential investor is able to appreciate the convenience of possible hedging strategies.

As the NPV is the main decision criterion in capital budgeting theory, the analysis of the parameters' relevance on NPV variability is fundamental. Any relative measure of worth should be consistent with NPV not only in terms of classical consistency but also in terms of output variability with respect to changes in the inputs.

Definition 4. (strong NPV-consistency) Given an analysis technique, a metric $\varphi$ (and its associated decision criterion) is strongly $N P V$-consistent (or NPV-compatible) if it fulfills Definition 2 and $N P V$ and $\varphi$ are coherent functions. The metric $\varphi$ is strictly $N P V$-consistent if the coherence is strict.

If a metric/criterion possesses strong NPV-consistency, the investor can equivalently adopt NPV or such criterion for measuring the value creation under uncertainty. In case a metric is not strongly NPV-consistent, the degree of incompatibility can be measured through Spearman's coefficient or through top-down coefficient, as seen in section 4.

We now show that, contrary to IRR, the SL-AIRR possesses strong NPV-consistency. To this end, we maintain the symbol $\alpha=\left(\alpha_{1}, \ldots, \alpha_{n}\right)$ as the vector of the project's value drivers (revenues, costs, interest, taxes, working capital, fixed assets etc.) and $\alpha^{0}$ is the base value. We assume that the initial invested capital (or borrowed amount) is exogenously given, as well as the $\mathrm{COC}$ (and $p$ ). The economic profitability of $P$ depends on the realization of the value drivers, which affect the FCFs, as seen in section $2: F_{t}=F_{t}(\alpha)$, $t=1,2, \ldots p$. We now let $f(\alpha)=\operatorname{NPV}(\alpha)=-c_{0}+\sum_{t=1}^{p} F_{t}(\alpha)(1+k)^{-t}$ and $g(\alpha)=\bar{\imath}(\alpha)$ be the SL-AIRR (as anticipated, we omit the dependence on $C^{S L}$ for simplicity).

Proposition 2. For any fixed $k, c_{0}$ and $p, S L-A I R R$ and NPV are strongly consistent in a strict sense under the following techniques:

(i) Standardized regression coefficient

(ii) Sensitivity Indices in variance-based decomposition models

(iii) Finite Change Sensitivity Indices

(iv) Helton's index

(v) Normalized Partial Derivative (NPD2 $\left.2_{i}^{f}\right)$

(vi) Differential Importance Measure.

Proof. The capital $C^{S L}$ does not depend on $\alpha$ so, using $(7), \bar{\imath}(\alpha)=q+l \cdot \operatorname{NPV}(\alpha)$ where $q=k$ and $l=(1+k) / C^{S L}$. The thesis follows from Proposition $1 .^{6}$

The above proposition guarantees that the value drivers' effect on the variability of SLAIRR and NPV is the same, not only in terms of ranks $\left(r^{\text {npv }}=r^{\bar{\imath}}\right)$ but also in terms of relevances $\left(R^{\mathrm{npv}}=R^{\bar{\imath}}\right)$. Therefore, $\rho_{\bar{\imath}, \mathrm{npv}}=\rho_{S^{\bar{\imath}}, S^{\mathrm{npv}}}=1$. This means that an investor can equivalently employ SL-AIRR or NPV to analyze an investment under uncertainty. By contrast, it should be evident that such a nice property is not satisfied by the IRR and, in general, it is not possible to determine an analytical relationship between NPV and IRR in a sensitivity analysis (see also Borgonovo and Peccati 2006, Percoco and Borgonovo 2012). Indeed, let $\alpha^{*} \in A$ be a given value of parameters and $x^{*}$ be the associated IRR, such that $\operatorname{NPV}\left(\alpha^{*}, x^{*}\right)=0 .{ }^{7}$ If there exists a neighbourhood of $\alpha^{*}$ where function $\operatorname{NPV}(\alpha, k)$ is a continuously differentiable function and $\operatorname{NPV}_{k}^{\prime}\left(\alpha^{*}, x^{*}\right) \neq 0$, then

\footnotetext{
${ }^{6}$ Evidently, SL-AIRR is strongly NPV-consistent under $N P D 1_{i}^{f}$ but not in a strict sense.

${ }^{7}$ Let $\alpha$ be a generic value belonging to a neighbourhood of $\alpha^{*}$. $\operatorname{NPV}(\alpha, k)$ is the $\mathrm{NPV}$ calculated with discount rate $k$.
} 
there exists a neighbourhood $V\left(\alpha^{*}\right) \subset A$ and a neighbourhood $W\left(x^{*}\right) \subset \mathbb{R}$ such that $x(\alpha): V \rightarrow W$ is the implicitly-defined function from the equation $\operatorname{NPV}(\alpha, k)=0$ and

$$
\begin{gathered}
x\left(\alpha^{*}\right)=x^{*}, \\
\operatorname{NPV}(\alpha, x(\alpha))=0, \forall \alpha \in V, \\
x_{a_{i}}^{\prime}(\alpha)=-\frac{\operatorname{NPV}_{a_{i}}^{\prime}(\alpha, x(\alpha))}{\operatorname{NPV}_{k}^{\prime}(\alpha, x(\alpha))}, \forall \alpha \in V .
\end{gathered}
$$

In particular,

$$
x_{a_{i}}^{\prime}\left(\alpha^{*}\right)=-\frac{\operatorname{NPV}_{a_{i}}^{\prime}\left(\alpha^{*}, x^{*}\right)}{\operatorname{NPV}_{k}^{\prime}\left(\alpha^{*}, x^{*}\right)} .
$$

\section{Some numerical examples}

In this section we will discuss a simple model, consisting of a firm facing the opportunity of investing in a 4-period project whose estimated revenues and costs are denoted as $R_{t}$ and $C_{t}$. We assume that clients pay in cash and suppliers are paid in cash, and also assume that the tax rate is zero, $\tau=0$. This implies $D e p_{t}=-\left(\Delta W C_{t}+\Delta N F A_{t}\right)$, whence $F_{t}=R_{t}-C_{t}-D e p_{t}-\left(\Delta W C_{t}+\Delta N F A_{t}\right)=R_{t}-C_{t}$. The project's value drivers are then $\alpha_{i}=R_{i}$ for $i=1,2,3,4$ and $\alpha_{i}=C_{i-4}$ for $i=5,6,7,8$. Hence, the value driver's vector is $\alpha=\left\{R_{1}, R_{2}, R_{3}, R_{4}, C_{1}, C_{2}, C_{3}, C_{4}\right\}$. NPV is computed as:

$$
\mathrm{NPV}(\alpha)=-c_{0}+\frac{R_{1}-C_{1}}{1+k}+\frac{R_{2}-C_{2}}{(1+k)^{2}}+\frac{R_{3}-C_{3}}{(1+k)^{3}}+\frac{R_{4}-C_{4}}{(1+k)^{4}} .
$$

Example 4. Assume $c_{0}=750$ and $k=10 \%$. Table 2 describes the base value $\alpha^{0}=$ $\left(R_{1}^{0}, R_{2}^{0}, R_{3}^{0}, R_{4}^{0}, C_{1}^{0}, C_{2}^{0}, C_{3}^{0}, C_{4}^{0}\right)$ and reports the corresponding Free Cash Flows and valuation metrics. The NPV is $157.37=-750+380 / 1.1+270 /(1.1)^{2}+360 /(1.1)^{3}+100 /(1.1)^{4}$. Considering that $750 / 4=187.5$, the vector of capitals associated with SL-AIRR is $c^{S L}=$ $(750,562.5,375,187.5,0)$ and $C^{S L}=1712.15=750+562.5 / 1.1+375 /(1.1)^{2}+187.5 /(1.1)^{3}$. Therefore SL-AIRR is equal to $\bar{\imath}=10 \%+157.37 / 1712.15 \cdot 1.1=20.11 \%$. The IRR exists and is unique: $x=20.86 \%$.

Table 2: Investment evaluated in $\alpha^{0}$

\begin{tabular}{lccccc}
\hline & 0 & 1 & 2 & 3 & 4 \\
\hline$R_{t}^{0}$ & & 580 & 570 & 560 & 400 \\
$C_{t}^{0}$ & & 200 & 300 & 200 & 300 \\
$F_{t}$ & -750 & 380 & 270 & 360 & 100 \\
\hline Valuation & & & & & \\
\hline NPV & 157.37 & & & & \\
$\bar{\imath}$ & $20.11 \%$ & & & & \\
$x$ & $20.86 \%$ & & & & \\
\hline
\end{tabular}

We now illustrate some sensitivity analysis' applications to NPV, SL-AIRR and IRR focusing on two techniques: FCSI and DIM. It will turn out that, while the SL-AIRR are strictly coherent, the degree of incoherence between IRR and NPV can be rather high.

\subsection{IRR versus SL-AIRR using FCSIs}

We show three numerical applications. In the first one, IRR is unique but is not strongly NPV-consistent. In the second one, despite IRR exists and is unique in $\alpha^{0}$, it does not exist in $\alpha^{1}$, making it impossible to perform an SA with IRR. In the third one, multiple IRRs arise for $\alpha=\alpha^{1}$; hence, the SA with IRR is ambiguous. By contrast, SL-AIRR 
exists, is unique, and is strongly NPV-consistent (in a strict sense) in each of the three cases.

Example 5. Consider project $P$ described in Example 4. The new value of revenues and costs $\alpha^{1}$ is shown in Table 3, with the corresponding new value of $F_{t}$, NPV, SLAIRR and IRR. In $\alpha^{1} \mathrm{NPV}$ is 442.92 , SL-AIRR is $38.46 \%$, IRR is $41.12 \%$ (it exists and is unique). The observed variations are: $\Delta \mathrm{NPV}=285.55=442.92-157.37 ; \Delta \bar{\imath}=18.35 \%=$ $38.46 \%-20.11 \% ; \Delta x=20.25 \%=41.12 \%-20.86 \%$. Table 4 shows cash flows, NPV, SL-AIRR and IRR calculated in $\left(\alpha_{i}^{1}, \alpha_{(-i)}^{0}\right)$; each line corresponds to the variation from $\alpha^{0}$ to $\alpha^{1}$ of a specific parameter, with the remaining parameters equal to the base value $\alpha^{0}$. Table 5 shows the individual effects $\left(\Delta_{i} f\right)$, the First Order FCSIs $\left(\Phi_{i}^{1, f}\right)$, the ranks $\left(r_{i}^{f}\right)$ and the Savage Scores of parameters $\left(S_{i}^{f}\right)$ for NPV, SL-AIRR and IRR.

The First Order FCSIs of NPV and SL-AIRR are equal: $\Phi_{i}^{1, \mathrm{npv}}=\Phi_{i}^{1, \bar{\imath}}$. SL-AIRR and NPV are strongly coherent in a strict sense . Evidently the parameters' ranking of NPV and SL-AIRR is equal, and $\rho_{\bar{\imath}, \mathrm{npv}}=\rho_{S^{\bar{\imath}}, S^{\mathrm{npv}}}=1$.

The First Order FCSIs of NPV and IRR are different: $\Phi_{i}^{1, \text { npv }} \neq \Phi_{i}^{1, x}$. NPV ranking and IRR ranking of parameters are also different. Therefore, IRR is not NPV-consistent according to Definition 4. The degree of inconsistency may be measured via (one minus) Spearman's coefficient or top-down coefficient: $1-\rho_{x, \mathrm{npv}}=1-0.857=0.143$ and $1-$ $\rho_{S^{x}, S^{\mathrm{npv}}}=1-0.77=0.23$ are the degrees of incompatibility of NPV and IRR according to the two alternative measures.

Table 6 shows cash flows, NPV, SL-AIRR and IRR corresponding to $\left(\alpha_{i}^{0}, \alpha_{(-i)}^{1}\right)$; in each line a specific parameter is fixed to the base value $\alpha^{0}$, while the remaining parameters are kept equal to the new value $\alpha^{1}$. Table 7 shows total effects $\left(\Delta_{i}^{T} f\right)$, Total Order FCSIs $\left(\Phi_{i}^{T, f}\right)$, ranks $\left(r_{i}^{f}\right)$ and Savage scores $\left(S_{i}^{f}\right)$ in functions $\operatorname{NPV}(\alpha), \bar{\imath}(\alpha)$, and $x(\alpha)$. The Total Order FCSIs of NPV and SL-AIRR are equal: $\Phi_{i}^{T, \text { npv }}=\Phi_{i}^{T, \bar{\imath}}$. SL-AIRR and NPV are strictly coherent.

The Total Order FCSIs of NPV and IRR are different: $\Phi_{i}^{T, \text { npv }} \neq \Phi_{i}^{T, x}$. The parameters' ranking differs as well. It is clear that, even in such a simple example, IRR's inconsistency is not negligible: The degree of inconsistency using the Spearman's coefficient is 1 $\rho_{x, \text { npv }}=1-0.667=0.333$ and is even greater if top-down coefficient is used: $1-\rho_{S^{x}, S^{\text {npv }}}=$ $1-0.409=0.591$.

Table 3: Investment evaluated in $\alpha^{1}$

\begin{tabular}{lccccc}
\hline & 0 & 1 & 2 & 3 & 4 \\
\hline$R_{t}$ & & 800 & 810 & 780 & 630 \\
$C_{t}$ & & 350 & 250 & 380 & 600 \\
$F_{t}$ & -750 & 450 & 560 & 400 & 30 \\
\hline Valuation & & & & & \\
\hline NPV & 442.92 & & & & \\
$\bar{\imath}$ & $38.46 \%$ & & & & \\
$x$ & $41.12 \%$ & & & & \\
\hline
\end{tabular}

Example 6. Consider a project $P$ such that $c_{0}=750$ and $k=10 \%$. Therefore $C^{S L}=$ 1712.15. The base value is $\alpha^{0}=(630,740,850,600,180,390,490,550)$; the new value is $\alpha^{1}=(600,700,800,500,200,400,500,850) . \alpha^{1}$ is a worse scenario than $\alpha^{0}$, with lower revenues and higher costs. Table 8 shows the project's cash flows and the corresponding NPV, SL-AIRR and IRR in $\alpha^{0}$ and $\alpha^{1}$. In the base case, IRR exists and is unique and it is equal to $28.52 \%$. In $\alpha^{1}$ IRR does not exist. This implies that the sensitivity analysis cannot be applied for IRR in $\alpha^{1}: \Delta x$ is not defined, hence the First Order FCSIs of IRR are not calculable; the total effects of parameters on IRR are not defined either. Therefore, the Total Order FCSIs of IRR do not exist. 
Table 4: Cash flows, NPV, SL-AIRR and IRR in $\left(\alpha_{i}^{1}, \alpha_{(-i)}^{0}\right)$

\begin{tabular}{lcccccccc}
\hline & $F_{0}$ & $F_{1}$ & $F_{2}$ & $F_{3}$ & $F_{4}$ & NPV & $\bar{\imath}$ & $x$ \\
\hline$\left(R_{1}^{1}, \alpha_{\left(-R_{1}\right)}^{0}\right)$ & -750 & 600 & 270 & 360 & 100 & 357.37 & $32.96 \%$ & $37.02 \%$ \\
$\left(R_{2}^{1}, \alpha_{\left(-R_{2}\right)}^{0}\right)$ & -750 & 380 & 510 & 360 & 100 & 355.72 & $32.85 \%$ & $33.84 \%$ \\
$\left(R_{3}^{1}, \alpha_{\left(-R_{3}\right)}^{0}\right)$ & -750 & 380 & 270 & 580 & 100 & 322.66 & $30.73 \%$ & $30.09 \%$ \\
$\left(R_{4}^{1}, \alpha_{\left(-R_{4}\right)}^{0}\right)$ & -750 & 380 & 270 & 360 & 330 & 314.46 & $30.20 \%$ & $28.49 \%$ \\
$\left(C_{1}^{1}, \alpha_{\left(-C_{1}\right)}^{0}\right)$ & -750 & 230 & 270 & 360 & 100 & 21.01 & $11.35 \%$ & $11.36 \%$ \\
$\left(C_{2}^{1}, \alpha_{\left(-C_{2}\right)}^{0}\right)$ & -750 & 380 & 320 & 360 & 100 & 198.69 & $22.77 \%$ & $23.63 \%$ \\
$\left(C_{3}^{1}, \alpha_{\left(-C_{3}\right)}^{0}\right)$ & -750 & 380 & 270 & 180 & 100 & 22.13 & $11.42 \%$ & $11.70 \%$ \\
$\left(C_{4}^{1}, \alpha_{\left(-C_{4}\right)}^{0}\right)$ & -750 & 380 & 270 & 360 & -200 & -47.53 & $6.95 \%$ & $5.30 \%$ \\
\hline
\end{tabular}

Table 5: First Order FCSI

\begin{tabular}{|c|c|c|c|c|c|c|c|c|c|c|c|c|}
\hline \multirow[b]{2}{*}{ Parameter } & \multicolumn{4}{|c|}{$\mathrm{NPV}$} & \multicolumn{4}{|c|}{ SL-AIRR } & \multicolumn{4}{|c|}{ IRR } \\
\hline & $\Delta_{i} \mathrm{NPV}$ & $\Phi_{i}^{1, \mathrm{npv}}$ & $r_{i}^{\mathrm{npv}}$ & $S_{i}^{\text {npv }}$ & $\Delta_{i} \bar{\imath}$ & $\Phi_{i}^{1, \bar{\imath}}$ & $r_{i}^{\bar{i}}$ & $S_{i}^{\bar{\imath}}$ & $\Delta_{i} x$ & $\Phi_{i}^{1, x}$ & $r_{i}^{x}$ & $S_{i}^{x}$ \\
\hline$R_{1}$ & 200.00 & $70.04 \%$ & 2 & 1.718 & $12.85 \%$ & $70.04 \%$ & 2 & 1.718 & $16.16 \%$ & $79.78 \%$ & 1 & 2.718 \\
\hline$R_{2}$ & 198.35 & $69.46 \%$ & 3 & 1.218 & $12.74 \%$ & $69.46 \%$ & 3 & 1.218 & $12.97 \%$ & $64.05 \%$ & 3 & 1.218 \\
\hline$R_{3}$ & 165.29 & $57.89 \%$ & 4 & 0.885 & $10.62 \%$ & $57.89 \%$ & 4 & 0.885 & $9.23 \%$ & $45.56 \%$ & 5 & 0.635 \\
\hline$R_{4}$ & 157.09 & $55.01 \%$ & 5 & 0.635 & $10.09 \%$ & $55.01 \%$ & 5 & 0.635 & $7.63 \%$ & $37.68 \%$ & 7 & 0.268 \\
\hline$C_{1}$ & -136.36 & $-47.76 \%$ & 6 & 0.435 & $-8.76 \%$ & $-47.76 \%$ & 6 & 0.435 & $-9.51 \%$ & $-46.93 \%$ & 4 & 0.885 \\
\hline$C_{2}$ & 41.32 & $14.47 \%$ & 8 & 0.125 & $2.65 \%$ & $14.47 \%$ & 8 & 0.125 & $2.77 \%$ & $13.68 \%$ & 8 & 0.125 \\
\hline$C_{3}$ & -135.24 & $-47.36 \%$ & 7 & 0.268 & $-8.69 \%$ & $-47.36 \%$ & 7 & 0.268 & $-9.16 \%$ & $-45.25 \%$ & 6 & 0.435 \\
\hline$C_{4}$ & -204.90 & $-71.76 \%$ & 1 & 2.718 & $-13.16 \%$ & $-71.76 \%$ & 1 & 2.718 & $-15.56 \%$ & $-76.83 \%$ & 2 & 1.718 \\
\hline \multicolumn{13}{|c|}{ Correlations } \\
\hline$\rho_{\bar{\imath}, \mathrm{npv}}$ & 1 & & & & & & & & & & & \\
\hline$\rho_{S^{\bar{\tau}}, S^{\mathrm{npv}}}$ & 1 & & & & & & & & & & & \\
\hline$\rho_{x, \mathrm{npv}}$ & 0.857 & & & & & & & & & & & \\
\hline$\rho_{S^{x}, S^{\mathrm{npv}}}$ & 0.770 & & & & & & & & & & & \\
\hline
\end{tabular}

Table 6: Cash flows, NPV, SL-AIRR and IRR in $\left(\alpha_{i}^{0}, \alpha_{(-i)}^{1}\right)$

\begin{tabular}{lccccrccc}
\hline & $F_{0}$ & $F_{1}$ & $F_{2}$ & $F_{3}$ & \multicolumn{1}{c}{$F_{4}$} & NPV & $\bar{\imath}$ & $x$ \\
\hline$\left(R_{1}^{0}, \alpha_{\left(-R_{1}\right)}^{1}\right)$ & -750 & 230 & 560 & 400 & 30 & 242.92 & $25.61 \%$ & $25.77 \%$ \\
$\left(R_{2}^{0}, \alpha_{\left(-R_{2}\right)}^{1}\right)$ & -750 & 450 & 320 & 400 & 30 & 244.57 & $25.71 \%$ & $27.89 \%$ \\
$\left(R_{3}^{0}, \alpha_{\left(-R_{3}\right)}^{1}\right)$ & -750 & 450 & 560 & 180 & 30 & 277.63 & $27.84 \%$ & $32.05 \%$ \\
$\left(R_{4}^{0}, \alpha_{\left(-R_{4}\right)}^{1}\right)$ & -750 & 450 & 560 & 400 & -200 & 285.82 & $28.36 \%$ & $34.21 \%$ \\
$\left(C_{1}^{0}, \alpha_{\left(-C_{1}\right)}^{1}\right)$ & -750 & 600 & 560 & 400 & 30 & 579.28 & $47.22 \%$ & $52.82 \%$ \\
$\left(C_{2}^{0}, \alpha_{\left(-C_{2}\right)}^{1}\right)$ & -750 & 450 & 510 & 400 & 30 & 401.59 & $35.80 \%$ & $38.45 \%$ \\
$\left(C_{3}^{0}, \alpha_{\left(-C_{3}\right)}^{1}\right)$ & -750 & 450 & 560 & 580 & 30 & 578.15 & $47.14 \%$ & $47.45 \%$ \\
$\left(C_{4}^{0}, \alpha_{\left(-C_{4}\right)}^{1}\right)$ & -750 & 450 & 560 & 400 & 330 & 647.82 & $51.62 \%$ & $48.19 \%$ \\
\hline
\end{tabular}


Table 7: Total Order FCSI

\begin{tabular}{|c|c|c|c|c|c|c|c|c|c|c|c|c|}
\hline \multirow[b]{2}{*}{ Parameter } & \multicolumn{4}{|c|}{ NPV } & \multicolumn{4}{|c|}{ SL-AIRR } & \multicolumn{4}{|c|}{ IRR } \\
\hline & $\Delta_{i}^{T} \mathrm{NPV}$ & $\Phi_{i}^{T, \mathrm{npv}}$ & $r_{i}^{\mathrm{npv}}$ & $S_{i}^{\mathrm{npv}}$ & $\Delta_{i}^{T} \bar{\imath}$ & $\Phi_{i}^{T, \bar{\imath}}$ & $r_{i}^{\bar{\imath}}$ & $S_{i}^{\bar{\imath}}$ & $\Delta_{i}^{T} x$ & $\Phi_{i}^{T, x}$ & $r_{i}^{x}$ & $S_{i}^{x}$ \\
\hline$R_{1}$ & 200.00 & $70.04 \%$ & 2 & 1.718 & $12.85 \%$ & $70.04 \%$ & 2 & 1.718 & $15.35 \%$ & $75.79 \%$ & 1 & 2.718 \\
\hline$R_{2}$ & 198.35 & $69.46 \%$ & 3 & 1.218 & $12.74 \%$ & $69.46 \%$ & 3 & 1.218 & $13.23 \%$ & $65.33 \%$ & 2 & 1.718 \\
\hline$R_{3}$ & 165.29 & $57.89 \%$ & 4 & 0.885 & $10.62 \%$ & $57.89 \%$ & 4 & 0.885 & $9.07 \%$ & $44.78 \%$ & 4 & 0.885 \\
\hline$R_{4}$ & 157.09 & $55.01 \%$ & 5 & 0.635 & $10.09 \%$ & $55.01 \%$ & 5 & 0.635 & $6.91 \%$ & $34.09 \%$ & 6 & 0.435 \\
\hline$C_{1}$ & -136.36 & $-47.76 \%$ & 6 & 0.435 & $-8.76 \%$ & $-47.76 \%$ & 6 & 0.435 & $-11.70 \%$ & $-57.78 \%$ & 3 & 1.218 \\
\hline$C_{2}$ & 41.32 & $14.47 \%$ & 8 & 0.125 & $2.65 \%$ & $14.47 \%$ & 8 & 0.125 & $2.67 \%$ & $13.18 \%$ & 8 & 0.125 \\
\hline$C_{3}$ & -135.24 & $-47.36 \%$ & 7 & 0.268 & $-8.69 \%$ & $-47.36 \%$ & 7 & 0.268 & $-6.34 \%$ & $-31.29 \%$ & 7 & 0.268 \\
\hline$C_{4}$ & -204.90 & $-71.76 \%$ & 1 & 2.718 & $-13.16 \%$ & $-71.76 \%$ & 1 & 2.718 & $-7.07 \%$ & $-34.93 \%$ & 5 & 0.635 \\
\hline \multicolumn{13}{|c|}{ Correlations } \\
\hline$\rho_{\bar{\imath}, \mathrm{npv}}$ & 1 & & & & & & & & & & & \\
\hline$\rho_{S^{\bar{\imath}}, S^{\mathrm{npv}}}$ & 1 & & & & & & & & & & & \\
\hline$\rho_{x, \mathrm{npv}}$ & 0.667 & & & & & & & & & & & \\
\hline$\rho_{S^{x}, S^{\mathrm{npv}}}$ & 0.409 & & & & & & & & & & & \\
\hline
\end{tabular}

SL-AIRR always exists and is unique, therefore it does not suffer from this problem. Table 9 shows the Total Order FCSIs of NPV and SL-AIRR: As expected, SL-AIRR and NPV are strongly coherent in a strict from (the First Order and Total Order FCSIs of NPV are equal; the same applies to SL-AIRR. Therefore only the Total Order Indices are reported).

Table 8: IRR not existing in $\alpha^{1}$

\begin{tabular}{lcc}
\hline & $\alpha^{0}$ & $\alpha^{1}$ \\
\hline$F_{0}$ & -750 & -750 \\
$F_{1}$ & 450 & 400 \\
$F_{2}$ & 350 & 300 \\
$F_{3}$ & 360 & 300 \\
$F_{4}$ & 50 & -350 \\
\hline Valuation & $\alpha^{0}$ & $\alpha^{1}$ \\
\hline NPV & 252.97 & -152.09 \\
$\bar{\imath}$ & $26.25 \%$ & $0.23 \%$ \\
$x$ & $28.52 \%$ & - \\
\hline
\end{tabular}

Example 7. Consider a project $P$, with $c_{0}=600$ and $k=8 \%$. Therefore $C^{S L}=$ 1392.94. The base value is $\alpha^{0}=(780,830,720,710,390,380,700,700)$; the new value is $\alpha^{1}=(750,800,710,680,400,370,500,830)$. The two scenarios are quite similar, the main difference is the costs' allocation in periods 3 and 4 . Table 10 shows cash flows, NPV, SL-AIRR and IRR in $\alpha^{0}$ and $\alpha^{1}$. In $\alpha^{0}$ the IRR function supplies a unique value and is equal to $26.96 \%$, the Hotelling's capital is $C^{x}=969.29$, therefore IRR is an investment rate. For $\alpha^{1}$ there exist two different IRRs: $x_{1}\left(\alpha^{1}\right)=25.18 \%, x_{2}\left(\alpha^{1}\right)=-61.75 \%$, so the sensitivity analysis is problematic: It is not clear which one IRR should be the relevant one.

Note also that the Hotelling value corresponding to $x_{1}\left(\alpha^{1}\right)$ is positive $\left(C^{0.2518}=\right.$ 937.98), so $x_{1}\left(\alpha^{1}\right)=25.181 \%$ is an investment rate; the Hotelling value corresponding to $x_{2}\left(\alpha^{1}\right)$ is negative $\left(C^{-0.6175}=-231.00\right)$, hence $x_{2}\left(\alpha^{1}\right)=-61.75 \%$ is a borrowing rate. Therefore, a change in the value drivers vector may cause IRR to change financial nature (from investment rate to borrowing rate and viceversa). SL-AIRR does not suffer from this kind of problems, because it always exists, is unique, and its financial nature only depends on the sign of $c_{0}$ (not on cost of capital nor on the value of parameters). In this case, SL-AIRR is an investment rate, regardless of the change in the value drivers. 
Table 9: IRR not existing in $\alpha^{1}$ : Total Order FCSI

\begin{tabular}{|c|c|c|c|c|c|c|}
\hline \multirow[b]{2}{*}{ Parameter } & \multicolumn{2}{|c|}{ NPV } & \multicolumn{2}{|c|}{ SL-AIRR } & \multicolumn{2}{|c|}{ IRR } \\
\hline & $\Phi_{i}^{T, \text { npv }}$ & $r_{i}^{\mathrm{npv}}$ & $\Phi_{i}^{T, \bar{\imath}}$ & $\overline{r_{i}^{\bar{\imath}}}$ & $\Phi_{i}^{T, x}$ & $\overline{r_{i}^{x}}$ \\
\hline$R_{1}$ & $6.73 \%$ & 5 & $6.73 \%$ & 5 & - & - \\
\hline$R_{2}$ & $8.16 \%$ & 4 & $8.16 \%$ & 4 & - & - \\
\hline$R_{3}$ & $9.27 \%$ & 3 & $9.27 \%$ & 3 & - & - \\
\hline$R_{4}$ & $16.86 \%$ & 2 & $16.86 \%$ & 2 & - & - \\
\hline$C_{1}$ & $4.49 \%$ & 6 & $4.49 \%$ & 6 & - & - \\
\hline$C_{2}$ & $2.04 \%$ & 7 & $2.04 \%$ & 7 & - & - \\
\hline$C_{3}$ & $1.85 \%$ & 8 & $1.85 \%$ & 8 & - & - \\
\hline$C_{4}$ & $50.59 \%$ & 1 & $50.59 \%$ & 1 & - & - \\
\hline
\end{tabular}

Table 11 shows the Total Order FCSIs of NPV and SL-AIRR: As now obvious, SLAIRR and NPV are strongly coherent in strict sense.

Table 10: Multiple IRR in $\alpha^{1}$

\begin{tabular}{lcc}
\hline & $\alpha^{0}$ & $\alpha^{1}$ \\
\hline$F_{0}$ & -600 & -600 \\
$F_{1}$ & 390 & 350 \\
$F_{2}$ & 450 & 430 \\
$F_{3}$ & 20 & 210 \\
$F_{4}$ & 10 & -150 \\
\hline Valuation & $\alpha^{0}$ & $\alpha^{1}$ \\
\hline NPV & 170.14 & 149.18 \\
$\bar{\imath}$ & $21.19 \%$ & $19.57 \%$ \\
$x$ & $26.96 \%$ & $25.18 \% ;-61.75 \%$ \\
\hline
\end{tabular}

Table 11: Multiple IRR in $\alpha^{1}$ : Total Order FCSI

\begin{tabular}{|c|c|c|c|c|c|c|}
\hline \multirow[b]{2}{*}{ Parameter } & \multicolumn{2}{|l|}{$\mathrm{NPV}$} & \multicolumn{2}{|c|}{ SL-AIRR } & \multicolumn{2}{|c|}{ IRR } \\
\hline & $\Phi_{i}^{T, \mathrm{npv}}$ & $r_{i}^{\mathrm{npv}}$ & $\Phi_{i}^{T, \bar{\imath}}$ & $\overline{r_{i}^{\bar{\imath}}}$ & $\overline{\Phi_{i}^{T, x}}$ & $\overline{r_{i}^{x}}$ \\
\hline$R_{1}$ & $132.52 \%$ & 3 & $132.52 \%$ & 3 & - & - \\
\hline$R_{2}$ & $122.71 \%$ & 4 & $122.71 \%$ & 4 & - & - \\
\hline$R_{3}$ & $37.87 \%$ & 8 & $37.87 \%$ & 8 & - & - \\
\hline$R_{4}$ & $105.20 \%$ & 5 & $105.20 \%$ & 5 & - & - \\
\hline$C_{1}$ & $44.17 \%$ & 6 & $44.17 \%$ & 6 & - & - \\
\hline$C_{2}$ & $-40.90 \%$ & 7 & $-40.90 \%$ & 7 & - & - \\
\hline$C_{3}$ & $-757.46 \%$ & 1 & $-757.46 \%$ & 1 & - & - \\
\hline$C_{4}$ & $455.88 \%$ & 2 & $455.88 \%$ & 2 & - & - \\
\hline
\end{tabular}

\subsection{IRR versus SL-AIRR using DIMs}

The DIM technique is a local SA technique, so it measures the value drivers' importance on the objective function in a neighbourhood of $\alpha^{0}$. We assume that changes in the inputs are proportional, so the DIM is described in eq. (20). In particular, the first partial derivatives of $\operatorname{NPV}(\alpha)$, evaluated in $\alpha^{0}$, are

$$
\mathrm{NPV}_{\alpha_{i}}^{\prime}\left(\alpha^{0}\right)= \begin{cases}(1+k)^{-i}, & i=1,2,3,4 \\ -(1+k)^{-(i-4)}, & i=5,6,7,8\end{cases}
$$

The first partial derivatives of SL-AIRR, evaluated in $\alpha^{0}$, are

$$
\bar{\imath}_{\alpha_{i}}^{\prime}\left(\alpha^{0}\right)=\mathrm{NPV}_{\alpha_{i}}^{\prime}\left(\alpha^{0}\right) \cdot \frac{(1+k)}{C^{S L}} .
$$


The partial derivative of $\operatorname{NPV}(\alpha, k)$ with respect to $k$ is

$$
\mathrm{NPV}_{k}^{\prime}(\alpha, k)=-\frac{R_{1}-C_{1}}{(1+k)^{2}}-2 \cdot \frac{R_{2}-C_{2}}{(1+k)^{3}}-3 \cdot \frac{R_{3}-C_{3}}{(1+k)^{4}}-4 \cdot \frac{R_{4}-C_{4}}{(1+k)^{5}}
$$

and

$$
\mathrm{NPV}_{k}^{\prime}\left(\alpha^{0}, x^{0}\right)=-\frac{R_{1}^{0}-C_{1}^{0}}{\left(1+x^{0}\right)^{2}}-2 \cdot \frac{R_{2}^{0}-C_{2}^{0}}{\left(1+x^{0}\right)^{3}}-3 \cdot \frac{R_{3}^{0}-C_{3}^{0}}{\left(1+x^{0}\right)^{4}}-4 \cdot \frac{R_{4}^{0}-C_{4}^{0}}{\left(1+x^{0}\right)^{5}} .
$$

From (24) and (25)

$$
x_{\alpha_{i}}^{\prime}\left(\alpha^{0}\right)= \begin{cases}-\left(1+x^{0}\right)^{-i} \cdot\left(\mathrm{NPV}_{k}^{\prime}\left(\alpha^{0}, x^{0}\right)\right)^{-1}, & i=1,2,3,4 \\ \left(1+x^{0}\right)^{-(i-4)} \cdot\left(\mathrm{NPV}_{k}^{\prime}\left(\alpha^{0}, x^{0}\right)\right)^{-1}, & i=5,6,7,8\end{cases}
$$

We illustrate two numerical applications of DIM technique where IRR and NPV are not coherent according to Definition 4. It is evident that SL-AIRR is, again, strongly coherent in a strict form with NPV.

Example 8. We consider an investment $P$, with $c_{0}=900$ and $k=8 \%$. Therefore $C^{S L}=2089.41$. The base value is $\alpha^{0}=(900,1000,1100,1200,600,700,800,900)$. The corresponding cash-flow vector is $F=(-900,300,300,300,300)$ and $\operatorname{NPV}\left(\alpha^{0}\right)=93.64$, $\bar{\imath}\left(\alpha^{0}\right)=12.84 \%, x\left(\alpha^{0}\right)=12.59 \%$. Table 12 shows the DIMs, the ranks and the Savage scores. The DIMs for NPV and IRR are different: $D I M_{i}^{\text {npv }}\left(\alpha^{0}\right) \neq D I M_{i}^{x}\left(\alpha^{0}\right)$. Not even the ranking is equal; for example, $R_{1}$ has rank 4 for NPV, while it has rank 1 for IRR; $R_{4}$ has rank 1 for NPV, while it has rank 4 for IRR. IRR and NPV are not coherent according to Definition 4. As $1-\rho_{x, \mathrm{npv}}=0.262$ and $1-\rho_{S^{x}, S^{\mathrm{npv}}}=0.691$, the NPV-inconsistency of IRR is remarkable, especially when using top-down coefficient.

Example 9. We consider a project $P$ with $c_{0}=1000$ and $k=8 \%$. The base value $\alpha^{0}$ is described in Table 13. In $\alpha^{0}$, NPV $=463.81, \bar{\imath}=29.58 \%$ and $x=26.65 \%$ (IRR exists and is unique). The sensitivity analysis' results are reported in the aforementioned Table. SLAIRR and NPV are strictly coherent. IRR and NPV, instead, are not coherent according to Definition $4.1-\rho_{x, \mathrm{npv}}=0.286$ signals a rather high incompatibility between IRR and

\begin{tabular}{|c|c|c|c|c|c|c|c|c|c|c|}
\hline \multirow[b]{2}{*}{ Parameter } & \multirow[b]{2}{*}{$\alpha^{0}$} & \multicolumn{3}{|c|}{ NPV } & \multicolumn{3}{|c|}{ SL-AIRR } & \multicolumn{3}{|c|}{ IRR } \\
\hline & & $D I M_{i}^{\mathrm{npv}}\left(\alpha^{0}\right)$ & $r_{i}^{\mathrm{npv}}$ & $S_{i}^{\text {npv }}$ & $D I M_{i}^{\bar{\imath}}\left(\alpha^{0}\right)$ & $r_{i}^{\bar{\imath}}$ & $\overline{S_{i}^{\bar{\tau}}}$ & $D I M_{i}^{x}\left(\alpha^{0}\right)$ & $r_{i}^{x}$ & $S_{i}^{x}$ \\
\hline$R_{1}$ & 900 & $83.87 \%$ & 4 & 0.885 & $83.87 \%$ & 4 & 0.885 & $88.82 \%$ & 1 & 2.718 \\
\hline$R_{2}$ & 1000 & $86.28 \%$ & 3 & 1.218 & $86.28 \%$ & 3 & 1.218 & $87.65 \%$ & 2 & 1.718 \\
\hline$R_{3}$ & 1100 & $87.88 \%$ & 2 & 1.718 & $87.88 \%$ & 2 & 1.718 & $85.64 \%$ & 3 & 1.218 \\
\hline$R_{4}$ & 1200 & $88.77 \%$ & 1 & 2.718 & $88.77 \%$ & 1 & 2.718 & $82.97 \%$ & 4 & 0.885 \\
\hline$C_{1}$ & 600 & $-55.91 \%$ & 8 & 0.125 & $-55.91 \%$ & 8 & 0.125 & $-59.21 \%$ & 8 & 0.125 \\
\hline$C_{2}$ & 700 & $-60.40 \%$ & 7 & 0.268 & $-60.40 \%$ & 7 & 0.268 & $-61.36 \%$ & 7 & 0.268 \\
\hline$C_{3}$ & 800 & $-63.91 \%$ & 6 & 0.435 & $-63.91 \%$ & 6 & 0.435 & $-62.28 \%$ & 5 & 0.635 \\
\hline$C_{4}$ & 900 & $-66.58 \%$ & 5 & 0.635 & $-66.58 \%$ & 5 & 0.635 & $-62.23 \%$ & 6 & 0.435 \\
\hline \multicolumn{11}{|c|}{ Correlations } \\
\hline$\rho_{\bar{\imath}, \mathrm{npv}}$ & 1 & & & & & & & & & \\
\hline$\rho_{S^{\bar{\imath}}, S^{\mathrm{npv}}}$ & 1 & & & & & & & & & \\
\hline$\rho_{x, \mathrm{npv}}$ & 0.738 & & & & & & & & & \\
\hline$\rho_{S^{x}, S^{\mathrm{npv}}}$ & 0.309 & & & & & & & & & \\
\hline
\end{tabular}
NPV, which is even higher if one uses the top-down coefficient: $1-\rho_{S^{x}, S^{\mathrm{npv}}}=0.423$.

Table 12: Coherence under DIM technique (Example 8) 
Table 13: Coherence under DIM technique (Example 9)

\begin{tabular}{|c|c|c|c|c|c|c|c|c|c|c|}
\hline \multirow[b]{2}{*}{ Parameter } & \multirow[b]{2}{*}{$\alpha^{0}$} & \multicolumn{3}{|c|}{ NPV } & \multicolumn{3}{|c|}{ SL-AIRR } & \multicolumn{3}{|c|}{ IRR } \\
\hline & & 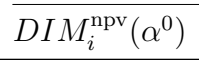 & $r_{i}^{\mathrm{npv}}$ & $S_{i}^{\mathrm{npv}}$ & $\overline{D I M_{i}^{\bar{\imath}}\left(\alpha^{0}\right)}$ & $r_{i}^{\bar{\imath}}$ & $S_{i}^{\bar{\imath}}$ & $\overline{D I M_{i}^{x}\left(\alpha^{0}\right)}$ & $r_{i}^{x}$ & $S_{i}^{x}$ \\
\hline$R_{1}$ & 700 & $44.28 \%$ & 3 & 1.218 & $44.28 \%$ & 3 & 1.218 & $55.27 \%$ & 1 & 2.718 \\
\hline$R_{2}$ & 780 & $45.68 \%$ & 1 & 2.718 & $45.68 \%$ & 1 & 2.718 & $48.63 \%$ & 2 & 1.718 \\
\hline$R_{3}$ & 810 & $43.93 \%$ & 4 & 0.885 & $43.93 \%$ & 4 & 0.885 & $39.87 \%$ & 3 & 1.218 \\
\hline$R_{4}$ & 900 & $45.19 \%$ & 2 & 1.718 & $45.19 \%$ & 2 & 1.718 & $34.98 \%$ & 4 & 0.885 \\
\hline$C_{1}$ & 300 & $-18.98 \%$ & 8 & 0.125 & $-18.98 \%$ & 8 & 0.125 & $-23.69 \%$ & 5 & 0.635 \\
\hline$C_{2}$ & 340 & $-19.91 \%$ & 6 & 0.435 & $-19.91 \%$ & 6 & 0.435 & $-21.20 \%$ & 6 & 0.435 \\
\hline$C_{3}$ & 380 & $-20.61 \%$ & 5 & 0.635 & $-20.61 \%$ & 5 & 0.635 & $-18.70 \%$ & 7 & 0.268 \\
\hline$C_{4}$ & 390 & $-19.58 \%$ & 7 & 0.268 & $-19.58 \%$ & 7 & 0.268 & $-15.16 \%$ & 8 & 0.125 \\
\hline \multicolumn{11}{|c|}{ Correlations } \\
\hline$\rho_{\bar{i}, \mathrm{npv}}$ & 1 & & & & & & & & & \\
\hline$\rho_{S^{\imath}, S^{n p v}}$ & 1 & & & & & & & & & \\
\hline$\rho_{x, \mathrm{npv}}$ & 0.714 & & & & & & & & & \\
\hline$\rho_{S^{x}, S^{\mathrm{npv}}}$ & 0.577 & & & & & & & & & \\
\hline
\end{tabular}

\section{Concluding remarks}

Many different investment criteria are available to managers, professionals and practitioners. NPV is considered a theoretically reliable measure of economic profitability. Industrial and financial investments are often evaluated through relative measures of worth as well. The most widely used relative measure is the IRR, which assumes that capital appreciates exponentially between two cash-flow dates and decreases, at the end of the period, by the distributed amount (or increases by the contributed amount). Recently, it has been introduced a new class of return rates named AIRR (Magni 2010, Magni 2013). Among these, this paper introduces the SL-AIRR, which assumes that the capital depreciates linearly (in a straight-line fashion).

Both IRR and SL-AIRR are coherent with NPV in the sense that both IRR criterion and SL-AIRR criterion correctly signal value creation or value destruction, just like the NPV (and, therefore, the decision made using one of the metrics is the same).

However, the IRR suffers from several difficulties, such as possible nonexistence or multiplicity. Conversely, SL-AIRR always exists and is unique. Furthermore, the financial nature of IRR (investment rate or borrowing rate) depends on cost of capital and, for a given initial capital amount, it depends on the project value drivers as well (revenues, costs, assets, tax rate, interest, etc.): A change in the value drivers may modify the financial nature of IRR or generate multiple IRRs with opposite financial nature. SLAIRR has an unambiguous financial nature, which is independent of both cost of capital and value drivers.

This work provides a new definition of NPV-consistency making use of sensitivity analysis (SA). Given an SA technique, a metric is strongly coherent with NPV if it fulfills the classical definition of NPV-consistency and generates the same ranking of the value drivers as that generated by the NPV. If, in addition, the parameters' relevances are equal to the ones associated with NPV, then the metric and NPV are strongly coherent in a strict form.

We assume that the COC is exogenously fixed by the decision maker, as well as the initial investment and the lifetime of the project. After proving that an affine transformation of a function preserves the ranking, we show that SL-AIRR, being an affine transformation of NPV, is strongly NPV-consistent under several (possibly, all) different techniques of SA.

On the contrary, it is not possible to determine a general relationship between IRR and NPV, since the IRR is an implicit function of NPV.

We have illustrated some simple numerical examples using FCSI (Borgonovo 2010a) 
and DIM (Borgonovo and Apostolakis 2001, Borgonovo and Peccati 2004), and have measured the degree of NPV-inconsistency of IRR via Spearman's (1904) coefficient and Iman and Conover's (1987) top-down coefficient. While SL-AIRR and NPV show perfect correlation, the incompatibility level between IRR and NPV can be remarkable. Furthermore, even when IRR exists and is unique in the base value, the value drivers' variation might be such that the sensitivity analysis is impossible (owing to inexistence or multiplicity of IRR).

The properties of SL-AIRR and IRR are summarized in the following table.

\begin{tabular}{lll}
\hline Property & SL-AIRR & IRR \\
\hline Classical NPV consistency & yes & yes \\
Existence & yes & no \\
Uniqueness & yes & no \\
Unambiguous financial nature & yes & no \\
Strong NPV consistency & yes & no \\
Strict NPV consistency & yes & no \\
\hline
\end{tabular}

This casts further shadows on the reliability of IRR as a relative measure of worth even in very simple cases. Most probably, more complex and realistic models might determine even higher incoherence levels between IRR and NPV: Further researches will be conducted for verifying this hypothesis. Conversely, the findings allow us to claim that the SL-AIRR can be reliably associated with NPV, providing consistent pieces of information. Also, the SL-AIRR is a good candidate for absolute NPV-consistency, to be intended as a perfect coherence under any possible technique of SA (this should hold, given the affine relation between SL-AIRR and NPV).

A further development of this work is the analysis of coherence between SL-AIRR and NPV versus the coherence of IRR and NPV in case COC is not exogenously fixed but is included in the set of value drivers. In this case, the relevance of COC for IRR is nihil, whereas both SL-AIRR and NPV are functions of the COC. Given the strong coherence between SL-AIRR and NPV for any given COC, one might expect that, even with uncertain cost of capital, the level of coherence between SL-AIRR and NPV is higher than the one between IRR and NPV (some preliminary analysis confirms this hypothesis).

\section{References}

Borgonovo, E. (2010a) Sensitivity analysis with finite changes: An application to modified EOQ models. European Journal of Operational Research, 200, 127-138.

Borgonovo, E. (2010b) A Methodology for Determining Interactions in Probabilistic Safety Assessment Models by Varying One Parameter at a Time. Risk Analysis, 30 (3), 385-399.

Borgonovo, E., Apostolakis, G. E. (2001) A new importance measure for risk-informed decision-making. Reliability Engineering and System Safety, 72 (2), 193-212.

Borgonovo, E., Gatti, S., Peccati, L. (2010) What drives value creation in investment projects? An application of sensitivity analysis to project finance transactions. European Journal of Operational Research, 205, 227-236.

Borgonovo, E., Peccati, L. (2004) Sensitivity analysis in investment project evaluation. International Journal of Production Economics, 90, 17-25.

Borgonovo, E., Peccati, L. (2006) Uncertainty and global sensitivity analysis in the evaluation of investment projects. International Journal of Production Economics, 104, 62-73. 
Borgonovo, E., Plischke, E. (2016) Sensitivity analysis: A review of recent advances. European Journal of Operational Research, 248(3), 869-887.

Borgonovo, E., Tarantola, S., Plischke, E., Morris, M.D. (2014) Transformations and invariance in the sensitivity analysis of computer experiments. Journal of the Royal Statistical Society, B, 76, Part 5, 925-947.

Brealey, R.A., Myers, S.C. (2000) Principles of Corporate Finance. McGraw-Hill, New York.

Bring, J. (1994) How to standardize regression coefficients. The American Statistician, 48(3)(August), 209-213.

Hazen, G.B. (2003) A new perspective on multiple internal rates of return. The Engineering Economist, 48(1), 31-51.

Helton, J.C. (1993) Uncertainty and sensitivity analysis techniques for use in performance assessment for radioactive waste disposal. Reliability Engineering and System Safety, 42, 327-367.

Iman, R.L., Conover, W.J. (1987) A measure of top-down correlation. Technometrics, 29 (3), 351-357.

Joy, O.M., Bradley, J.O. (1973) A note on sensitivity analysis of rates of return. Journal of Finance (December 1973), 1255-1261.

Magni, C.A. (2010) Average Internal Rate of Return and investment decisions: A new perspective. The Engineering Economist, 55(2), 150-180.

Magni, C.A. (2013) The Internal Rate of Return approach and the AIRR Paradigm: A refutation and a corroboration. The Engineering Economist: A Journal Devoted to the Problems of Capital Investment, 58(2), 73-111.

Percoco, M., Borgonovo, E. (2012) A note on the sensitivity analysis of the internal rate of return. International Journal of Production Economics, 135, 526-529.

Pianosi, F., Beven, F., Freer, J., Hall, J.W., Rougier, J., Stephenson, D.B., Wagener, T. (2016) Sensitivity analysis of environmental models: A systematic review with practical workflow. Environmental Modelling \& Software, 79, 214-232.

Rabitz, H., Alis, O.F. (1999) General foundations of high-dimensional model representations. Journal of Mathematical Chemistry, 25, 197-233.

Ross, S.A., Westerfield, R.W., Jordan, B.D. (2011) Essentials of corporate finance, 7th ed. McGraw-Hill/Irwin, New York.

Saltelli, A., Marivoet, J. (1990) Non-parametric statistics in sensitivity analysis for model output: A comparison of selected techniques. Reliability Engineering and System Safety, $28,229-253$.

Saltelli, A., Ratto, M., Andres, T., Campolongo, F., Cariboni, J., Gatelli, D., Saisana, M., Tarantola, S. (2008) Global Sensitivity Analysis. The Primer. John Wiley \& Sons. 
Saltelli, A., Tarantola, S., Campolongo, F., Ratto, M. (2004) Sensitivity analysis in practice. A guide to assessing scientific models. John Wiley \& Sons.

Savage, I.R. (1956) Contributions to the theory of rank order statistics - The two-sample case. The Annals of Mathematical Statistics, 27(3), 590-615.

Sobol', I.M. (1993) Sensitivity estimates for nonlinear mathematical models. Mathematical Modeling and Computational Experiments, 1(4), 407-414.

Sobol', I.M. (2001) Global sensitivity indices for nonlinear mathematical models and their Monte Carlo estimates. Mathematics and Computers in Simulation, 55, 271-280.

Spearman, C. (1904) The proof and measurement of association between two things. The American Journal of Psychology, 15(1), 72-101.

White, G.I., Sondhi, A.C., Fried, D. (1997) The Analysis and Use of Financial Statements. Second edition. Wiley, New York, USA. 